\title{
Energy cycle associated with inter-member variability in a large ensemble of simulations with the Canadian RCM (CRCM5)
}

\author{
Oumarou Nikiéma ${ }^{1} \cdot$ René Laprise $^{1}$
}

Received: 26 August 2014 / Accepted: 10 April 2015 / Published online: 10 May 2015

(C) The Author(s) 2015. This article is published with open access at Springerlink.com

\begin{abstract}
In an ensemble of Regional Climate Model (RCM) simulations where different members are initialised at different times but driven by identical lateral boundary conditions, the individual members provide different, but equally acceptable, weather sequences. In others words, RCM simulations exhibit the phenomenon of Internal Variability (or inter-member variability-IV), defined as the spread between members in an ensemble of simulations. Our recent studies reveal that RCM's IV is associated with energy conversions similar to those taking place in weather systems. By analogy with the classical work on global energetics of weather systems, a formulation of an energy cycle for IV has been developed that is applicable over limited-area domains. Prognostic equations for ensemble-mean kinetic energy and available enthalpy are decomposed into contributions due to ensemble-mean variables and those due to deviations from the ensemble mean (IV). Together these equations constitute an energy cycle for IV in ensemble simulations of an RCM. A 50-member ensemble of 1-year simulations that differ only in their initial conditions was performed with the fifth-generation Canadian RCM (CRCM5) over an eastern North America domain. The various energy reservoirs of IV and exchange terms between reservoirs were evaluated; the results show a remarkably close parallel between the energy conversions associated with IV in ensemble simulations of RCM and
\end{abstract}

Oumarou Nikiéma

oumarou.nikiema@uqam.ca

1 Département des Sciences de la Terre et de l'atmosphère, Centre ESCER (Étude et Simulation du Climat à l'Échelle Régionale), UQAM, B.P. 8888, Stn. Downtown, Montreal, QC H3C 3P8, Canada the energy conversions taking place in weather systems in the real atmosphere.

Keywords Regional climate models - Ensemble of simulations $\cdot$ Inter-member variability $\cdot$ Energy cycle

\section{List of symbols}

$a$

$a_{h}$

$a_{p}, a_{T}$

$a_{s}, a_{B}, a_{C}$

$A_{E M}$

$A_{E M B}, A_{E M S}, A_{E M C}$

$A_{I V}$

B

$C_{A}$

$C_{A B}, C_{A S}$

$C_{E M}$

$C_{E M B}, C_{E M S}, C_{E M B S}$
Average earth radius

Available enthalpy

Pressure and temperature components of available enthalpy

Stratification, baroclinic and cross-term components of available enthalpy

Available enthalpy of ensemble-mean fields Available enthalpy of ensemble-mean fields due to baroclinicity stratification and both effects

Available enthalpy of intermember variability

Pressure-dependent part of

$a_{h}$

Conversion of enthalpy energy between $A_{E M}$ and $A_{I V}$ Their sum is a result of $C_{A}$ : $\left[C_{A}=C_{A B}+C_{A S}\right]$

Conversion term into the ensemble-mean state Conversion term into the ensemble-mean state due to baroclinicity, stratification and both effects: 


$\begin{array}{llll} & {\left[C_{E M}=C_{E M B}+C_{E M S}+I_{A B}\right]} & R & \text { Gas constant for air } \\ C_{I V} & \text { Conversion term into the } & S & \text { Entropy } \\ & \text { deviation from ensemble- } & S_{r} & \text { Reference entropy } \\ & \text { mean state } & T & \text { Temperature } \\ & \text { Conversion of kinetic } & T_{r} & \text { Reference temperature value } \\ C_{K} & \text { energy between } K_{E M} \text { and } K_{I V} & \vec{V}(u, v) & \text { Horizontal wind vector } \\ & \text { Specific heat at constant } & z & \text { Altitude } \\ c_{p} & \text { pressure for dry air } & \alpha & \text { Specific volume } \\ & \text { Term associated with the } & \omega & \text { Vertical movement in pres- } \\ D_{E M} & \text { energy dissipation in the } E M & & \text { sure coordinate }(d p / d t) \\ & \text { state } & \Phi & \text { Geopotential height } \\ & \text { Term associated with the } & \varphi & \text { Latitude } \\ D_{I V} & \text { energy dissipation in the } I V & \theta & \text { Potential temperature } \\ & \text { state } & \psi & \text { General atmospheric } \\ & \text { Ensemble-mean } & & \text { parameter } \\ & \text { Horizontal momentum } & \langle\rangle & \text { Ensemble-mean operator } \\ F & \text { sources/sinks } & ()^{\prime} & \text { Deviation from } E M \\ F_{B}, F_{A_{E M}}, F_{A_{I V}}, F_{K_{I V}}, F_{K_{E M}}, & \text { Transport term for } B, A_{E M}, & () * & \text { Deviation from } T_{r} \\ & A_{I V}, K_{I V} \text { and } K_{E M} & ()^{\times} & \text {Deviation from horizon- } \\ F_{A_{E M B}}, F_{A_{E M S}} & \text { Transport term for } A_{E M B} \text { and } & & \text { tal average along isobaric } \\ g & A_{E M S} & & \text { surfaces } \\ G_{E M}, G_{I V} & \text { Gravitational acceleration } & () & \text { Horizontal average along } \\ & \text { Term associated with the } & & \text { isobaric surfaces }\end{array}$

$G_{E M B}, G_{E M S}$

H

$H_{A_{E M}}, H_{A_{I V}}, H_{K_{E M}}, H_{K_{I V}}$

$H_{A_{E M B}}, H_{A_{E M S}}$

$I_{A B}$

IV

$K$

$K_{I V}$

$K_{E M}$

$n$

$N$

$p_{s}, p_{T}$

$p$

$p_{r}$

$p_{00}$

$Q$ energy generated in the EM

and IV states

Term associated with the energy generated for $A_{E M B}$ and $A_{E M S}$ reservoirs

Enthalpy

Third-order terms of $A_{E M}$, $A_{I V}, K_{E M}, K_{I V}$ prognostic equations

Third-order terms of $A_{E M B}$, $A_{E M S}$ prognostic equations Conversion term between $A_{E M}$ and $B$

Inter-member variability

Kinetic energy

Kinetic energy for intermember variability

Kinetic energy of ensemble-mean Index number of the simulation in the ensemble Total number of simulations Pressure at bottom and top of atmosphere

Pressure

Reference value of pressure Standard value of pressure Total diabatic heating rate

\section{Introduction}

In climate modelling, ensemble simulations have become a standard approach to filter out the unpredictable component of the Earth system, to provide estimates of the uncertainties associated with climate projections and to improve the determination of rare events such as climate extremes (IPCC 2013). Regional Climate Models (RCM) are used to make retrospective climate simulations and future climate projections that include realistic weather sequences due to their capacity of representing the physical processes with higher resolution. RCM are integrated on a limited-area domain from initial conditions (IC) and lateral boundary conditions (LBC) provided either by an archived simulation of a driving Global Climate Model (GCM) or by gridded analyses of observations.

In numerical weather prediction, it is well known that repeated forecasts launched with even minute differences in initial conditions will eventually diverge from one another (Lorenz 1963). The same behaviour is noted in ensemble of climate model simulations, whether global or regional, which exhibit a kind of uncertainty called inter-member (or internal) variability (IV). IV can be defined as the spread between members in an ensemble of simulations that differ only in their IC. With global models the IV asymptotes to the transient-eddy variability in the limit of large 
ensembles. In the case of regional models, however, the LBC exert a constraint that limits the inter-member spread (at least at the large scales), which contributes to generally maintaining the magnitude of IV below the transient-eddy variability (e.g., Weisse et al. 2000; Giorgi and Bi 2000; Rinke and Dethloff 2000; Christensen et al. 2001; Caya and Biner 2004; Rinke et al. 2004; Lucas-Picher et al. 2004 and 2008a, b; Alexandru et al. 2007; Nikiéma and Laprise 2011a, b).

A remarkable behaviour is that the RCM's IV fluctuates in time with some episodic large growth. Previous studies, such as those of Giorgi and Bi (2000) and Alexandru et al. (2007), have shown that the IV is not associated with magnitude or nature of the IC differences. These studies converge to the same conclusion that the IV magnitude strongly fluctuates according to synoptic events in RCM simulations. It is also well documented that IV greatly varies with weather regime, season, domain size and model configuration. For instance, over mid-latitudes such as North America regional domain, it was found that IV increases with domain size and, for precipitation and geopotential height, stronger IV occurs in summer as a result of the combination of slower upper tropospheric winds, implying longer residence time within the regional domain and reduced control exerted by LBC on simulations, and stronger local forcing processes such as convection (Alexandru et al. 2007; Lucas-Picher et al. 2004, 2008a, b).

Alexandru et al. (2007) were amongst the first to study the IV climatology by using a large ensemble of RCM simulations with 20 members. They used the version 3.6.1 of the Canadian RCM (CRCM; Caya and Laprise 1999) to study the 1993 summer-season IV over eastern North America. Each simulation in the 20-member ensemble shared exactly the same LBC; the difference between them consisted only in a delay of $24 \mathrm{~h}$ in IC between each members. Their results showed that the IV magnitude strongly fluctuates with synoptic events during the simulations, and that the geographical distribution of IV differed between variables. They suggested that strong precipitation events in the southern United States appear to act as a triggering mechanism for the 850-hPa geopotential IV, which continues to develop along the storm track, reaching its maximum amplitude toward the north-east of their simulation domain.

Nikiéma and Laprise (2011a, b) extended the work of Alexandru et al. (2007) in order to shed some light on the physical processes responsible of large episodic growths of IV. They performed a quantitative diagnostic calculation to identify the various diabatic and dynamical contributions to the temporal variation and spatial distribution of IV. They established IV budget equations for the potential temperature and the relative vorticity, and they identified the terms responsible for the IV fluctuations. Their studies revealed that the dominant terms responsible for the episodic large increase of potential temperature IV are the covariance term involving the potential temperature fluctuations and diabatic heating fluctuations, and the covariance of intermember fluctuations acting upon ensemble-mean (EM) gradients. Their results also indicated that the episodes of large decreases of IV occur when maxima of IV approach the outflow boundary and are eventually transported out of the regional domain by the EM flow.

It was noted in these studies that the processes responsible for IV fluctuations in ensemble of RCM simulations closely parallel the energy conversions taking place in weather systems. Hence Nikiéma and Laprise (2013, hereafter referred to as NL13) proposed an approximate formulation of an energy cycle for IV, applicable on limited-area domains, which we will use to quantify the various contributions in an ensemble of CRCM5 simulations. In this paper, we use a large ensemble of 50-member simulations over 1 year to analyze various contributions of IV energy cycle proposed by NL13. The paper is structured as follows. Section 2 describes the model used and the simulation design, and recapitulates briefly the IV energy cycle equations. Results are presented in Sect. 3 and the main conclusions are summarized in Sect. 4.

\section{Model, data set and evaluation methods}

\subsection{Model description and simulation design}

The model used in the present study is the fifth-generation Canadian RCM (CRCM5) described in HernándezDíaz et al. (2013) and Martynov et al. (2013). For the present study, sea-surface temperature (SST) and sea-ice coverage are prescribed from Era-Interim Reanalyses (Kalnay et al. 1996) that also provide the atmospheric LBC for the model; these are linearly interpolated in time for each time step for the model. Although CRCM5 code offers the option of large-scale spectral nudging, this option was not used in order to allow the IV to fully develop.

This study uses a 50-member ensemble of simulations carried out on a domain of 300 by 200 grid points, with a grid mesh of $0.3^{\circ}$ and 56 terrain-following hybrid levels in the vertical. The free domain, excluding the lateral sponge zone and semi-Lagrangian halo, contains $260 \times 160$ grid points and it covers eastern North America and part of the Atlantic Ocean. All the simulations use the same LBC; the 
only difference between simulations is the initial time to start the simulations. Each member starts at 0000 UTC on different days from October 12th 2004 to November 30th 2004. Thus, fifty simulations were run for 1 year. The simulated data were archived at $3 \mathrm{~h}$ intervals, from December 1 st 2004 at 0300 UTC to December 1st 2005 at 0000 UTC; data will be analysed interpolation on the following set of 15 pressure levels: $50,70,100,150,200,250,300,400$, 500, 600, 700, 850, 925, 975 and $1000 \mathrm{hPa}$.

\subsection{Methodology: inter-member variability energy cycle}

Expanding upon previous studies on global atmospheric energetics pioneered by Lorenz $(1955,1967)$, Pearce (1978) and Marquet (1991) established an energy formalism that can be applied to atmospheric energetics over limited-area domains. NL13 applied a similar approach to establish an energy cycle for IV based on an ensemble of RCM simulations. In the following, we summarize the methodology and we refer the reader to NL13 for more details on the algebraic details.

Noting by $n$ the simulation member index in an ensemble of $N$ members, each atmospheric variable $\Psi_{n} \in\left\{T_{n}, u_{n}, v_{n}, \omega_{n}, \Phi_{n}, \ldots\right\}$ can be split in two components: an ensemble-mean (EM) part $\langle\Psi\rangle$ and deviation thereof $\Psi^{\prime}$ :

$\Psi=\langle\Psi\rangle+\Psi_{n}^{\prime}$

where the EM is calculated as:

$\langle\Psi\rangle=\frac{1}{N} \sum_{n=1}^{N} \Psi_{n}$

In the following the index $n$ will be left out without ambiguity. The IV is estimated as the EM of the deviation square:

$\sigma_{\Psi}^{2} \approx \frac{1}{N} \sum_{n=1}^{N} \Psi^{\prime 2} \equiv\left\langle\Psi^{\prime 2}\right\rangle$

The quadratic form of the kinetic energy $(K)$ leads to decompose its $\operatorname{EM}(\langle K\rangle)$ into two components as: $\langle K\rangle=K_{E M}+K_{I V}$

where $K_{E M}=\langle\vec{V}\rangle \cdot\langle\vec{V}\rangle / 2$ is the EM kinetic energy of the EM wind and $K_{I V}=\langle k\rangle / 2$ is the EM kinetic energy due to wind deviations from the EM, with $k=\overrightarrow{V^{\prime}} \cdot \overrightarrow{V^{\prime}}$.

Following Marquet (1991), the available enthalpy is decomposed in its temperature- and pressure-dependent components. The temperature-dependent component of the EM available enthalpy $\langle A\rangle$ is approximated as a quadratic expression and is further decomposed as

$\langle A\rangle=A_{E M}+A_{I V}$

where $A_{E M}=\frac{C_{p}}{2 T_{r}}\left\langle T-T_{r}\right\rangle^{2}$ is the EM enthalpy associated with the square of the EM temperature deviation from a reference temperature $T_{r}$, and $A_{I V}=\frac{C_{p}}{2 T_{r}}\left\langle T^{2}\right\rangle$ is the EM enthalpy due to the temperature deviations from the EM. In this formulation, $T_{r}$ is a constant reference temperature that is chosen so that its inverse corresponds to the time and space average of the inverse of $T$ over the domain of interest (Marquet 1991); here $T_{r}=260 \mathrm{~K}$ is used. The pressure-dependent component of the available enthalpy (B) is $B=R T_{r} \ln \left(p / p_{r}\right)$, which is a very simple term in pressure coordinates, with $p_{r}$ a reference pressure that depends on surface pressure $\left(p_{S}\right)$. It is noteworthy that the expressions of $A_{I V}$ and $K_{I V}$ are proportional to the inter-member variance of temperature and wind deviations, respectively.

From the basic field equations, NL13 have established the following approximate prognostic equations for $A_{I V}$, $K_{I V}, A_{E M}, K_{E M}$ and $B$ :

$L_{A_{I V}}=R_{A_{I V}}=G_{I V}+C_{A}-C_{I V}-F_{A_{I V}}-H_{A_{I V}}$

$L_{A_{E M}}=R_{A_{E M}}=G_{E M}+I_{A B}-C_{E M}-C_{A}-F_{A_{E M}}-H_{A_{E M}}$

$L_{K_{I V}}=R_{K_{I V}}=C_{I V}+C_{K}-D_{I V}-F_{K_{I V}}-H_{K_{I V}}$

$L_{K_{E M}}=R_{K_{E M}}=C_{E M}-C_{K}-D_{E M}-F_{K_{E M}}-H_{K_{E M}}$

$L_{B}=R_{B}=-F_{B}-I_{A B}$

where $L_{\mathrm{E}}=\partial E / \partial t$ is the tendency of the energy reservoirs $\mathrm{E} \in\left\{A_{I V}, A_{E M}, K_{I V}, K_{E M}, B\right\}$ and $R_{\mathrm{E}}$ is the sum of all contributions to the energy $\mathbf{E}$ budget: 
Fig. 1 The energy cycle associated with Inter-member Variability (IV) in ensemble of RCM simulations [reproduced with modifications from Nikiéma and Laprise (2013) () 2013 Springer-see Fig. 1]. The values represent the seasonal mean of different terms integrated over the whole regional domain

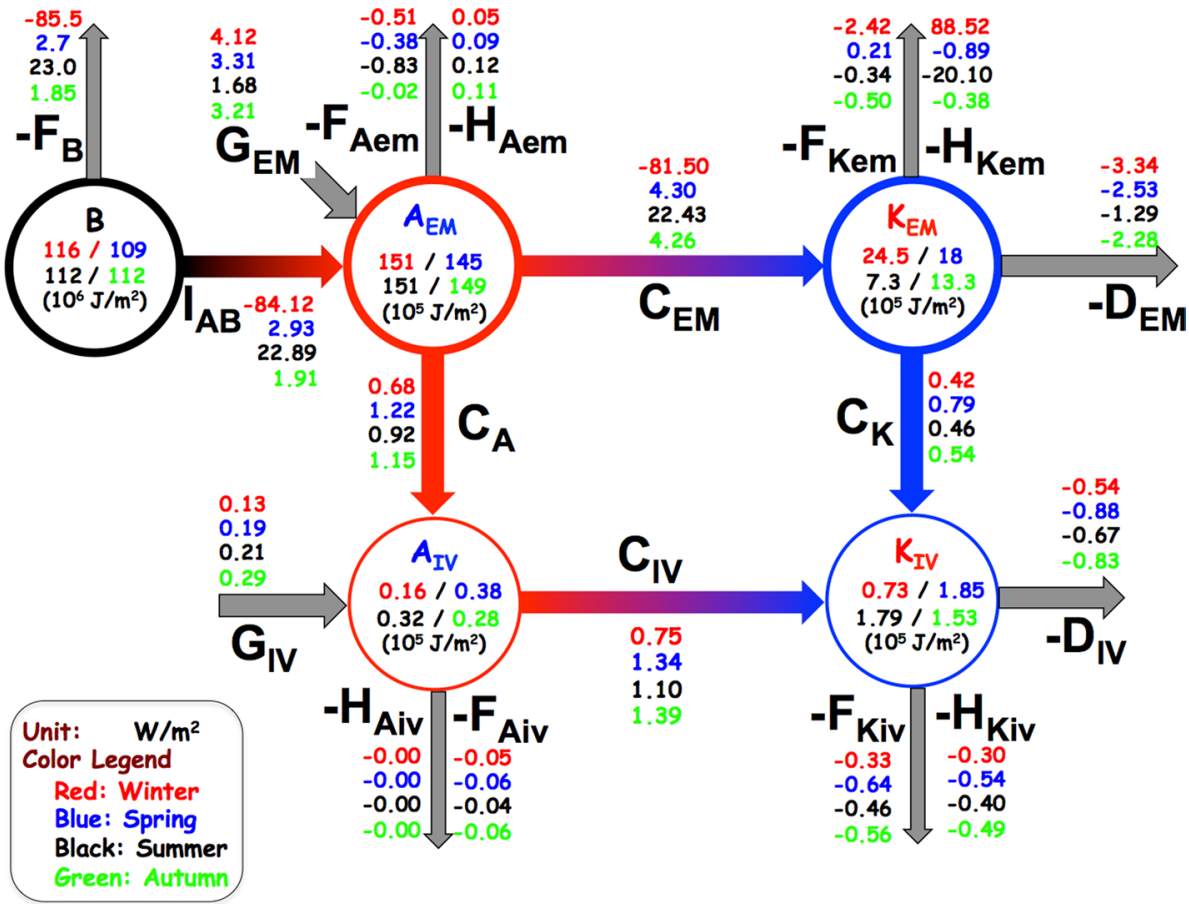

$$
\begin{aligned}
A_{I V}= & \frac{C_{p}}{2 T_{r}}\left\langle T^{\prime 2}\right\rangle ; \quad A_{E M}=\frac{C_{p}}{2 T_{r}}\left\langle T-T_{r}\right\rangle^{2} ; \quad K_{I V}=\left\langle\overrightarrow{V^{\prime}} \cdot \overrightarrow{V^{\prime}} / 2\right\rangle ; \quad K_{E M}=\langle\vec{V}\rangle \cdot\langle\vec{V}\rangle / 2 ; \quad B=R T_{r} \ln \left(\frac{p}{p_{r}}\right) \\
G_{I V}= & l\left\langle T^{\prime} Q^{\prime} / T_{r}\right\rangle ; \quad G_{E M}=\frac{l}{T_{r}}\left\langle T_{*}\right\rangle\langle Q\rangle ; \quad D_{I V}=-\left\langle\overrightarrow{V^{\prime}} \cdot \overrightarrow{F^{\prime}}\right\rangle ; \quad D_{E M}=-\langle\vec{V}\rangle \cdot\langle\vec{F}\rangle \\
C_{I V}= & -\left\langle\omega^{\prime} \alpha^{\prime}\right\rangle ; \quad C_{E M}=-\langle\omega\rangle\langle\alpha\rangle ; \quad I_{A B}=-\frac{R T_{r}}{p}\langle\omega\rangle \\
C_{A}= & -\frac{\left\langle\overrightarrow{V^{\prime}} T^{\prime}\right\rangle}{T_{r}} \cdot \vec{\nabla}\left\langle C_{p} T\right\rangle-\frac{\left\langle\omega^{\prime} T^{\prime}\right\rangle}{T_{r}} \frac{\partial\left\langle C_{p} T\right\rangle}{\partial p} ; \quad C_{K}=-\left\langle\overrightarrow{V^{\prime}} \cdot\left(\overrightarrow{V^{\prime}} \cdot \vec{\nabla}\right)\langle\vec{V}\rangle\right\rangle-\left\langle\overrightarrow{V^{\prime}} \cdot\left(\omega^{\prime} \frac{\partial\langle\vec{V}\rangle}{\partial p}\right)\right\rangle \\
F_{\mathrm{E}}= & \vec{\nabla} \cdot(\langle\vec{V}\rangle \mathrm{E})+\frac{\partial(\langle\omega\rangle \mathrm{E})}{\partial p} ; \quad \text { with E } \in\left\{A_{E M}, A_{I V}, K_{E M}, K_{I V}, B\right\} \\
H_{A_{I V}}= & \frac{C_{p}}{2 T_{r}}\left\langle\vec{\nabla} \cdot\left(\overrightarrow{V^{\prime}} T^{\prime 2}\right)+\frac{\partial\left(\omega^{\prime} T^{\prime 2}\right)}{\partial p}\right\rangle ; \quad H_{K_{I V}}=\vec{\nabla} \cdot\left\langle\left(k+\Phi^{\prime}\right) \vec{V}^{\prime}\right\rangle+\frac{\partial\left\langle\left(k+\Phi^{\prime}\right) \omega^{\prime}\right\rangle}{\partial p} \\
H_{K_{E M}}= & \vec{\nabla} \cdot\left(\langle\vec{V}\rangle \cdot\left\langle\vec{V}^{\prime} \overrightarrow{V^{\prime}}\right\rangle\right)+\vec{\nabla} \cdot(\langle\vec{V}\rangle\langle\Phi\rangle)+\frac{\partial}{\partial p}\left(\langle\vec{V}\rangle \cdot\left\langle\vec{V}^{\prime} \omega^{\prime}\right\rangle\right)+\frac{\partial}{\partial p}(\langle\omega\rangle\langle\Phi\rangle) \\
H_{A_{E M}}= & \frac{C_{p}}{T_{r}} \vec{\nabla} \cdot\left(\left\langle T_{*}\right\rangle\left\langle\vec{V}^{\prime} T_{*}^{\prime}\right\rangle\right)+\frac{C_{p}}{T_{r}} \frac{\partial\left(\left\langle T_{*}\right\rangle\left\langle\omega^{\prime} T_{*}^{\prime}\right\rangle\right)}{\partial p}
\end{aligned}
$$

where $l \approx T_{r} /\langle T\rangle$ is a factor of order unity according to our choice of $T_{r}$ value.

Together, Eqs. (5)-(9) form an energy cycle for IV in an ensemble of RCM simulations. Schematically, Fig. 1 shows the IV energy cycle that is composed of five reservoirs linked together by energy conversion terms $(C$ and $I)$ and "forced" by several boundary terms $(D, F, G$ and $H)$. The external reservoir $(B)$ appears due to the fact that mass is not constant over an open limited-area domain. The terms $G$ acts as generation term for $A_{E M}$ and $A_{I V}$, whereas kinetic energy is destroyed by dissipation terms $D$. Because the energy cycle is computed locally over a regional domain, additional boundary fluxes terms ( $F$ and $H$ ) are present; these would vanish upon integration over the entire globe. The different values in Fig. 1 represent the seasonal mean of different terms integrated over the regional study domain. These results will be discussed in the next section. This energy cycle can be extended to a further decomposition in horizontal average on pressure surfaces and deviation thereof. In this case, the IV energy cycle takes another shape with additional reservoirs and conversion terms (see NL13's Fig. 2). 
Fig. 2 Vertical profile of seasonal mean and horizontalaveraged on pressure surfaces of the total ensemble-mean (EM) Available Enthalpy a due to EM square deviations from reference temperature $\left(\mathrm{A}_{\mathrm{EM}}\right)$, b due to the mean stratification $\left(\mathrm{A}_{\mathrm{EMS}}\right)$, and $\mathbf{c}$ due to baroclinicity $\left(\mathrm{A}_{\mathrm{EMB}}\right)$, and $\mathbf{d}$ Kinetic Energy associated with the EM wind $\left(\mathrm{K}_{\mathrm{EM}}\right)$. Panel e shows the time evolution of the horizontal average and vertical integral from 50 to surface pressure of the following fields: the pressure-dependent part available enthalpy (B), the total EM Available Enthalpy $\left(\mathrm{A}_{\mathrm{EM}}\right)$ with $\mathrm{A}_{\mathrm{EM}}=\mathrm{A}_{\mathrm{EMS}}+\mathrm{A}_{\mathrm{EMB}}$, the $\mathrm{A}_{\mathrm{EM}}$ due to stratification $\left(\mathrm{A}_{\mathrm{EMS}}\right)$, the $\mathrm{A}_{\mathrm{EM}}$ due to baroclinicity $\left(\mathrm{A}_{\mathrm{EMB}}\right)$ and EM Kinetic Energy $\left(\mathrm{K}_{\mathrm{EM}}\right)$
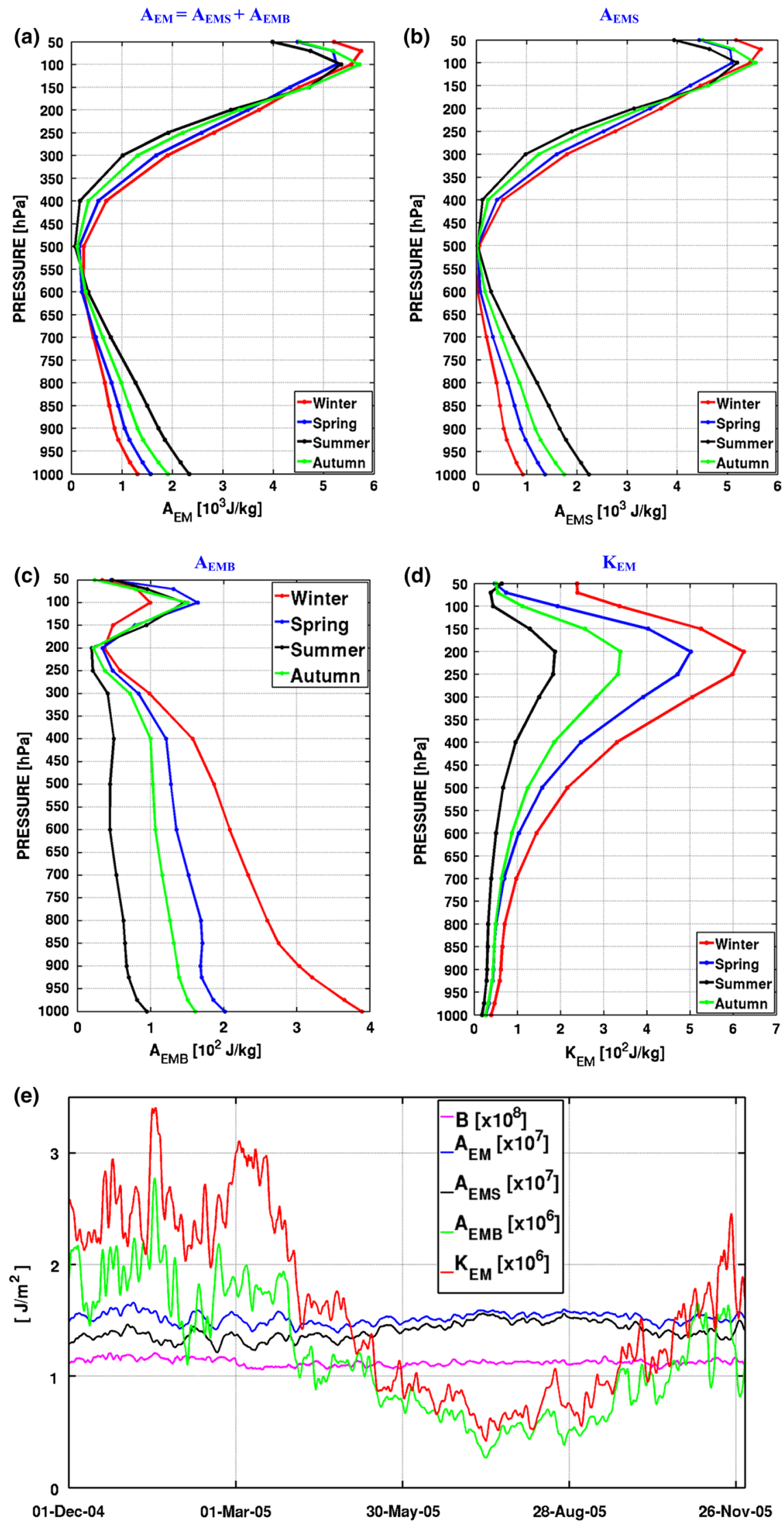
Fig. 3 Vertical profile of seasonal mean and horizontal-averaged on pressure surfaces of the a Inter-Member Variability (IV) Available Enthalpy $\left(\mathrm{A}_{\mathrm{IV}}\right)$ and $\mathbf{b}$ IV Kinetic Energy $\left(\mathrm{K}_{\mathrm{IV}}\right)$. Panel c shows the time evolutions of the horizontal average and vertical integral from 50 to surface pressure of $\mathrm{A}_{\mathrm{IV}}$ and $\mathrm{K}_{\mathrm{IV}}$
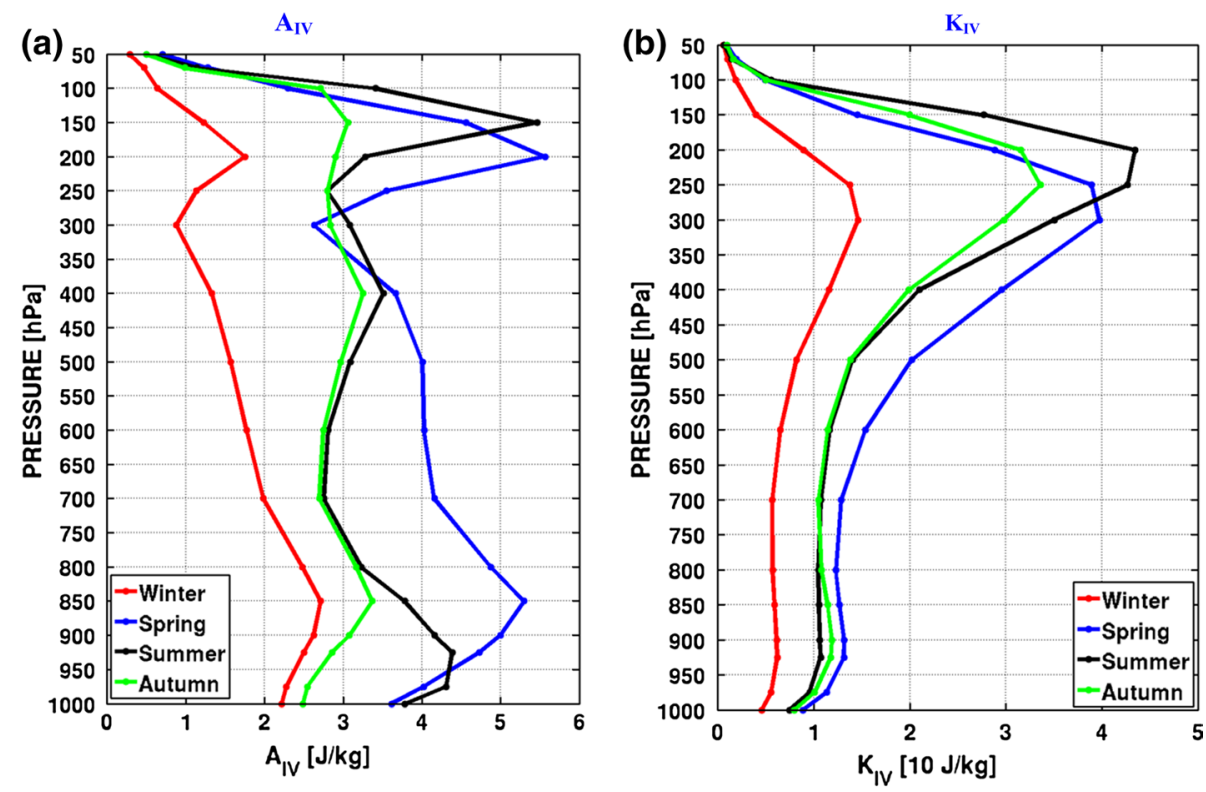

(c)

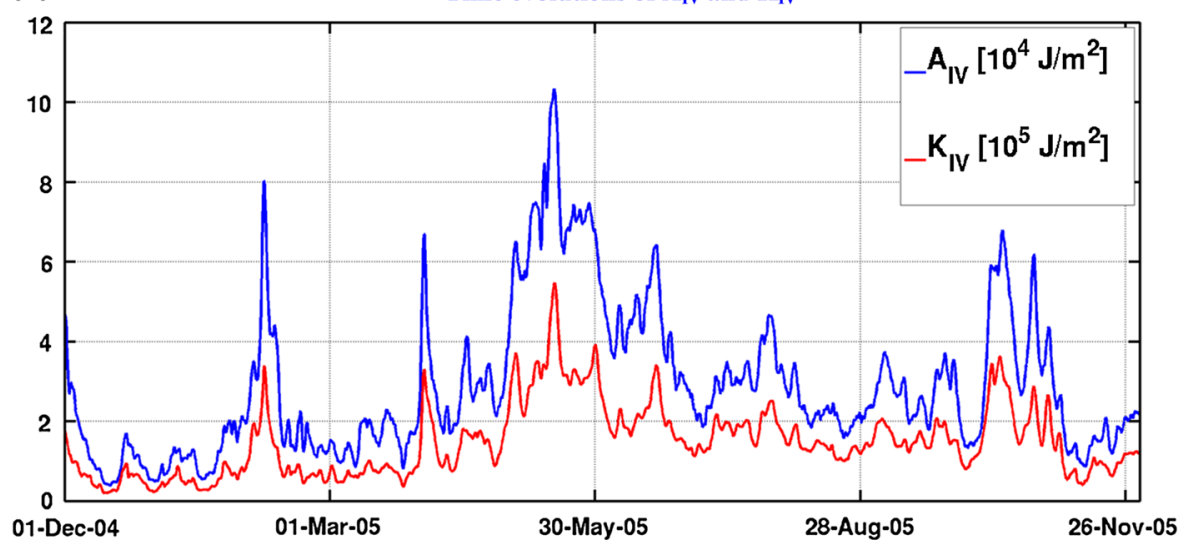

\section{Results and analysis}

\subsection{Available enthalpy and kinetic energy of ensemble-mean state}

Based on the 50 1-year simulations that differ only by their starting date, the available enthalpy and kinetic energy associated with the ensemble-mean state, $A_{E M}$ and $K_{E M}$, were computed following Eqs. (3) and (4). Figure 2a presents the vertical profile of seasonal mean of $A_{E M}$ averaged on pressure surfaces. The vertical profiles indicate a minimum at the middle troposphere (near the $500 \mathrm{hPa}$ level) where the temperature is, on average, close to the chosen value for the reference temperature $\left(T_{r}=260 \mathrm{~K}\right)$, and larger values of $A_{E M}$ appear in the upper and lower parts of the atmosphere. In order to overcome this no-physical representation of the available enthalpy, and following Marquet (1991), NL13 decomposed $A_{E M}$ into components due to baroclinicity $\left(A_{E M B}\right)$, mean stratification $\left(A_{E M S}\right)$, and a cross-term $\left(A_{E M C}\right)$ :

$A_{E M}=A_{E M B}+A_{E M S}+A_{E M C}$

where

$$
\begin{aligned}
& A_{E M B}=\frac{C_{p}}{2 T_{r}}\langle T-\bar{T}\rangle^{2} \\
& A_{E M S}=\frac{C_{p}}{2 T_{r}}\left\langle\bar{T}-T_{r}\right\rangle^{2} \\
& A_{E M C}=\frac{C_{p}}{2 T_{r}} 2\langle T-\bar{T}\rangle\left\langle\bar{T}-T_{r}\right\rangle
\end{aligned}
$$

where the over bar indicates the isobaric mean. After taking the average on pressure surfaces, the $\overline{A_{E M C}}$ term vanishes and the expression simplifies to

$\overline{A_{E M}}=\overline{A_{E M B}}+A_{E M S}$

where $\overline{A_{E M B}}=\frac{C_{p}}{2 T_{r}} \overline{\langle T-\bar{T}\rangle^{2}}$ and $A_{E M S}=\frac{C_{p}}{2 T_{r}}\left\langle\bar{T}-T_{r}\right\rangle^{2}$ 
Fig. 4 Seasonal average vertically integrated inter-member variability for a available enthalpy $\left(\mathrm{A}_{\mathrm{IV}}\right)$ and $\mathbf{b}$ kinetic energy $\left(K_{I V}\right)$

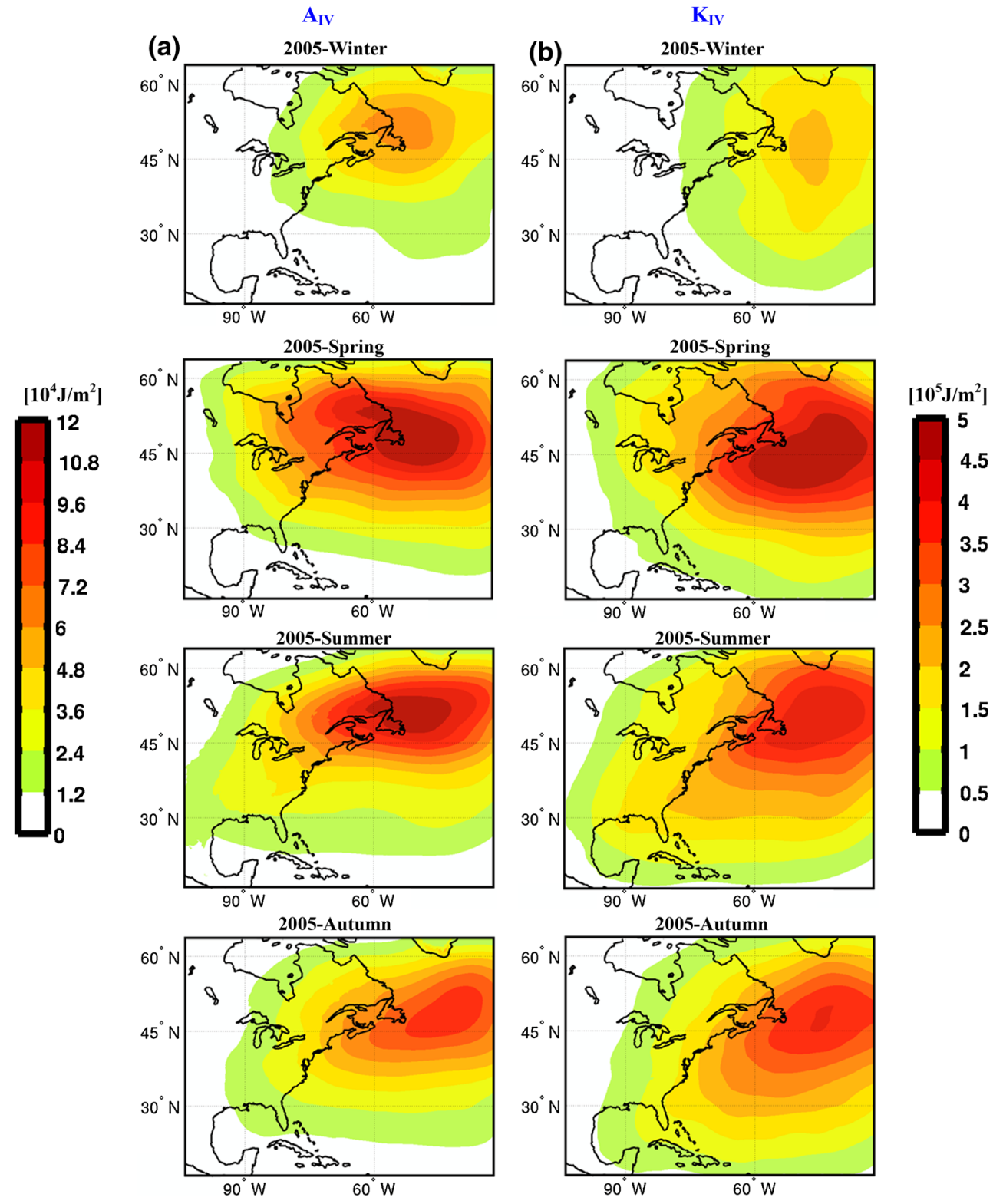

Figure $2 \mathrm{~b}$ and $\mathrm{c}$ show the vertical profiles of available enthalpy components due to mean stratification $\left(A_{E M S}\right)$ and baroclinicity $\left(\overline{A_{E M B}}\right)$, respectively. $A_{E M S}$ is the biggest reservoir of the total EM available enthalpy $\left(A_{E M}\right)$ and its vertical structure still reflects the choice of $T_{r}$. On the other hand, the baroclinic available enthalpy $\left(\overline{A_{E M B}}\right)$ has the same magnitude order as the EM kinetic energy $\left(K_{E M}\right)$ (panels $\mathrm{c}$ and $\mathrm{d}$ in Fig. 2; note the different scale used for different panels in Fig. 2), with values in around few hundred Joules per $\mathrm{kg}$. Figure $2 \mathrm{c}$ shows that $\overline{A_{E M B}}$ decreases with height in the troposphere, indicating that maximum gradients (i.e. differences between local and the isobaric-mean temperatures) are found near the ground. Similar profiles prevail in all seasons, but with different intensities; summer has the smallest values and winter the largest, and spring and autumn intermediate values, in agreement with known climatological features for this region of the world.

The kinetic energy of the ensemble-mean wind, $K_{E M}$, (panel $\mathrm{d}$ in Fig. 2) is maximum near the tropopause, reflecting the presence of the stronger upper-tropospheric winds associated with the jet stream. Again similar profiles of $K_{E M}$ prevail in all seasons, but with different intensities; summer has the smallest values and winter the largest, and spring and autumn intermediate values, in agreement with the known variations of the intensity of the jet stream. The agreement between the seasonal variations in the magnitudes of $A_{E M B}$ and $K_{E M}$ is a consequence of the thermalwind relation that prevails in mid-latitudes at large scales.

Figure 2e presents the time evolution of the different EM energies integrated over the whole domain. Results shows 
a remarkably similarity in the magnitude of $A_{E M B}$ and $K_{E M}$ as well as their time variations, with a correlation coefficient of about 0.93 ; this result partly reflects the fact that the wind and temperature fields satisfy the thermal-wind relationship. The larger values and time variations of $A_{E M B}$ and $K_{E M}$ occur in winter season due to large fluctuations of temperature and wind during this season in mid-latitude. On average in winter, $A_{E M B} \sim 1.8 \times 10^{6} \mathrm{~J} / \mathrm{m}^{2}$ and $K_{E M}$ $\sim 2.5 \times 10^{6} \mathrm{~J} / \mathrm{m}^{2}$. To get a physical sense of the meaning of these numbers, we note that a heat source of $11.6 \mathrm{~W} /$ $\mathrm{m}^{2}$ applied for 1 day results in an energy accumulation of $\sim 1 \times 10^{6} \mathrm{~J} / \mathrm{m}^{2}$. In summer these numbers decrease to about 6 and $8 \mathrm{~W} / \mathrm{m}^{2}$ per day, respectively. As shown in Fig. 2e, the contribution associated with the mean stratification $\left(A_{E M S}\right)$ is large, with values are around $1.4 \times 10^{7} \mathrm{~J} / \mathrm{m}^{2}$, but it exhibits little seasonal variation. The pressure-dependent part of available enthalpy is very large $\left(B \sim 10^{8} \mathrm{~J} / \mathrm{m}^{2}\right)$, but it varies little in time as a result of surface pressure variations.

\subsection{Available enthalpy and kinetic energy due to inter-member variability}

Figure 3 shows the vertical profiles of EM available enthalpy associated with inter-member variability of temperature $\left(A_{I V}\right.$, panel a) and EM kinetic energy associated with inter-member variability of winds $\left(K_{I V}\right.$, panel b), averaged horizontally on pressure surfaces over the study domain and in time for each seasons. The results indicate that IV contributions are smallest in winter and more important in spring and summer, and to a lesser extent in fall, confirming earlier results (e.g., Alexandru et al. 2007; Lucas-Picher et al. 2004, 2008a, b). It is noteworthy that the contributions from the EM fields shown earlier in Fig. 2 exhibited their largest amplitudes in winter.

Figure $3 \mathrm{c}$ shows the time evolution of the domainaveraged values of $A_{I V}$ and $K_{I V}$ (note however the different scales). One notes the strong synchronicity of the time variations of the two fields (time correlation of 0.96); to a good approximation, $K_{I V} \sim 5 A_{I V}$, reflecting the thermal-wind balance that prevails for the perturbations given that the atmosphere is in hydrostatic and quasi-geostrophic equilibrium (see "Appendix").

Figure 4 presents the maps of seasonal-mean vertically integrated fields of $A_{I V}$ and $K_{I V}$. Both variables show similar horizontal distributions at all seasons, with maximum IV energies in the northeast, outflow part of the study domain. The smallest values of IV energies occur in winter as noted before, in part because of the stronger advection of IV energy out of the domain by mean winds, resulting in stronger control exerted by LBC that limits the inter-member spread in the ensemble, while stronger local processes, such as condensation and convection, can explain the higher values of the IV in summer (Nikiéma and Laprise 2011a, b). Furthermore, results show that IV energies are also important in spring and autumn compared to those of summer (see Fig. 4). This result will be commented upon later in Sect. 3.3.1.

\subsection{Inter-member variability energy cycle in the ensemble of simulations}

We will now proceed to evaluate the contributions of the terms in the budget equations for the energy reservoirs $\mathrm{E} \in\left\{A_{E M}, A_{I V}, K_{E M}, K_{I V}, B\right\}$. Let us recall that in Eqs. (5)(9), the terms $L_{E}$ represent the time fluctuation of reservoirs of energy $\mathbf{E}$ in the 50-member ensemble of simulations; it is evaluated as a centred time difference of the energy $\mathbf{E}$ between two archival times $(3 \mathrm{~h})$ in the simulations. The terms $R_{\mathrm{E}}$ are the sum of all the contributions to the tendency of energy $\mathbf{E}$; they are calculated by evaluating numerically the terms in Eqs. (5)-(9), based on archived samples in the simulations, after their interpolation in pressure surfaces, and using numerical approximations for the derivatives as required.

The panels in Fig. 5 give an overview of the contributions of the various terms in the equations for the reservoirs $A_{I V}, K_{I V}, A_{E M}$ and $K_{E M}$; each dot represents the seasonal- and domain-averaged value of a term in the energy equations. One first notes that, as expected, both $L_{\mathrm{E}}$ and $R_{\mathrm{E}}$ are small, but not quite identical. In principle, the budget should close, $L_{\mathrm{E}}=R_{\mathrm{E}}$, but in practice the equality is only approximately satisfied due to the numerous discretization approximations and time sampling. A detailed analysis revealed that the time variations of $L_{\mathrm{E}}$ and $R_{\mathrm{E}}$ are highly correlated $(C \geq 0.95)$. Hence, the sum of incoming arrows for each IV energy reservoir corresponds approximately to the sum of outgoing arrows in Fig. 1.

Figure 5a indicates that, on average, the most important source of $A_{I V}$ is the conversion term $C_{A}$, followed by the diabatic generation term $G_{I V}$, while the conversion term $C_{I V}$ acts as a sink with similar magnitude as $C_{A}$, but of opposite sign. The other terms contribute little in a domain-averaged sense. Figure $5 \mathrm{~b}$ indicates that, on average, the conversion terms $C_{I V}$ and $C_{K}$ contribute positively to the tendency of $K_{I V}$, while the dissipation term $D_{I V}$ and boundary flux terms $F_{K I V}$ and $H_{K I V}$ contribute negatively. Figure 5c shows the various contributions to the $A_{E M}$ budget: the terms $G_{E M}$ and $I_{A B}$ generally act as sources, except $I_{A B}$ that acts as sink in winter, and the term $C_{E M}$ acts as sink for all seasons, except in winter when it acts as a source that counteracts the sink due to $I_{A B}$. The other terms have negligible contributions in a domain-averaged sense. Figure $5 \mathrm{~d}$ shows the different contributions to the $K_{E M}$ budget: the term $C_{E M}$ is the dominant source of $K_{E M}$, except in winter when it acts as a sink that is offset by a positive contribution by $-H_{K E M}$. 
Fig. 5 Time and domain averages of the different contributions to tendencies of $\mathbf{a} \mathrm{A}_{\mathrm{IV}}, \mathbf{b}$ $\mathrm{K}_{\mathrm{IV}}, \mathbf{c} \mathrm{A}_{\mathrm{EM}}$ and $\mathbf{d} \mathrm{K}_{\mathrm{EM}}$, for each season as indicated by dots of different colours: black for winter, red for spring, green for summer and blue for autumn. Note that some large-amplitude contributions have been scaled by a factor (as indicated in parenthesis) to keep the dot within range of the graphic scale
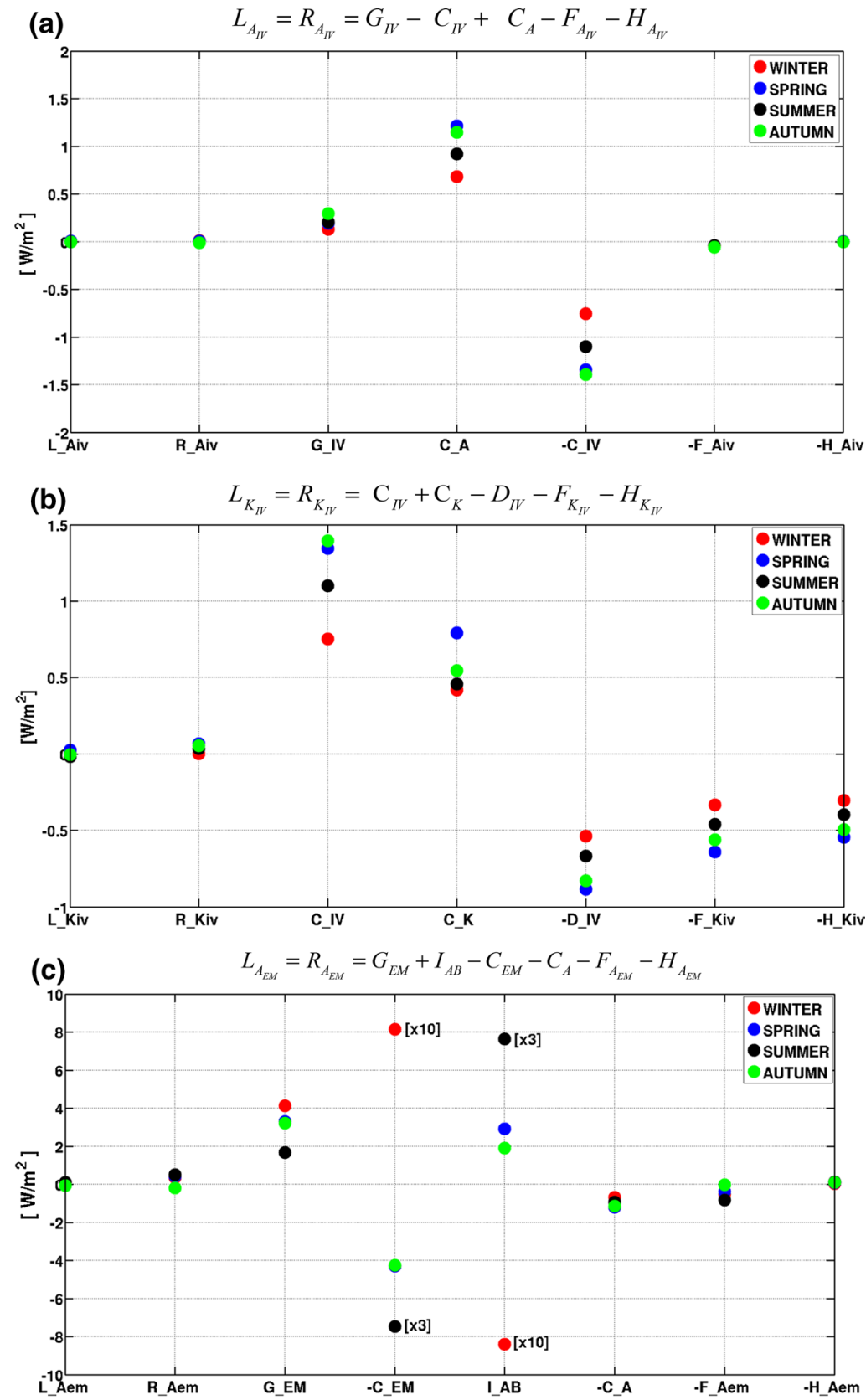

(d) $\quad L_{K_{E M}}=R_{K_{E M}}=C_{E M}-C_{K}-D_{E M}-F_{K_{E M}}-H_{K_{E M}}$

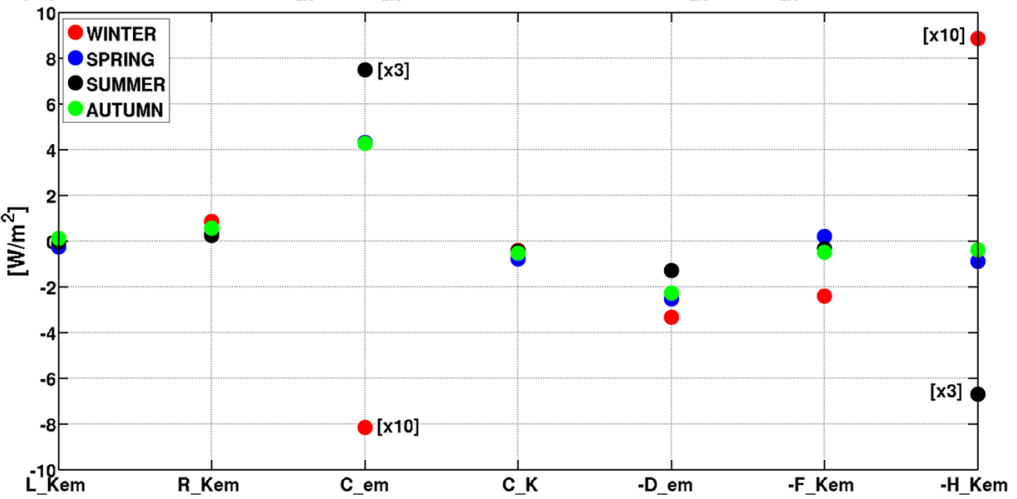


(a)

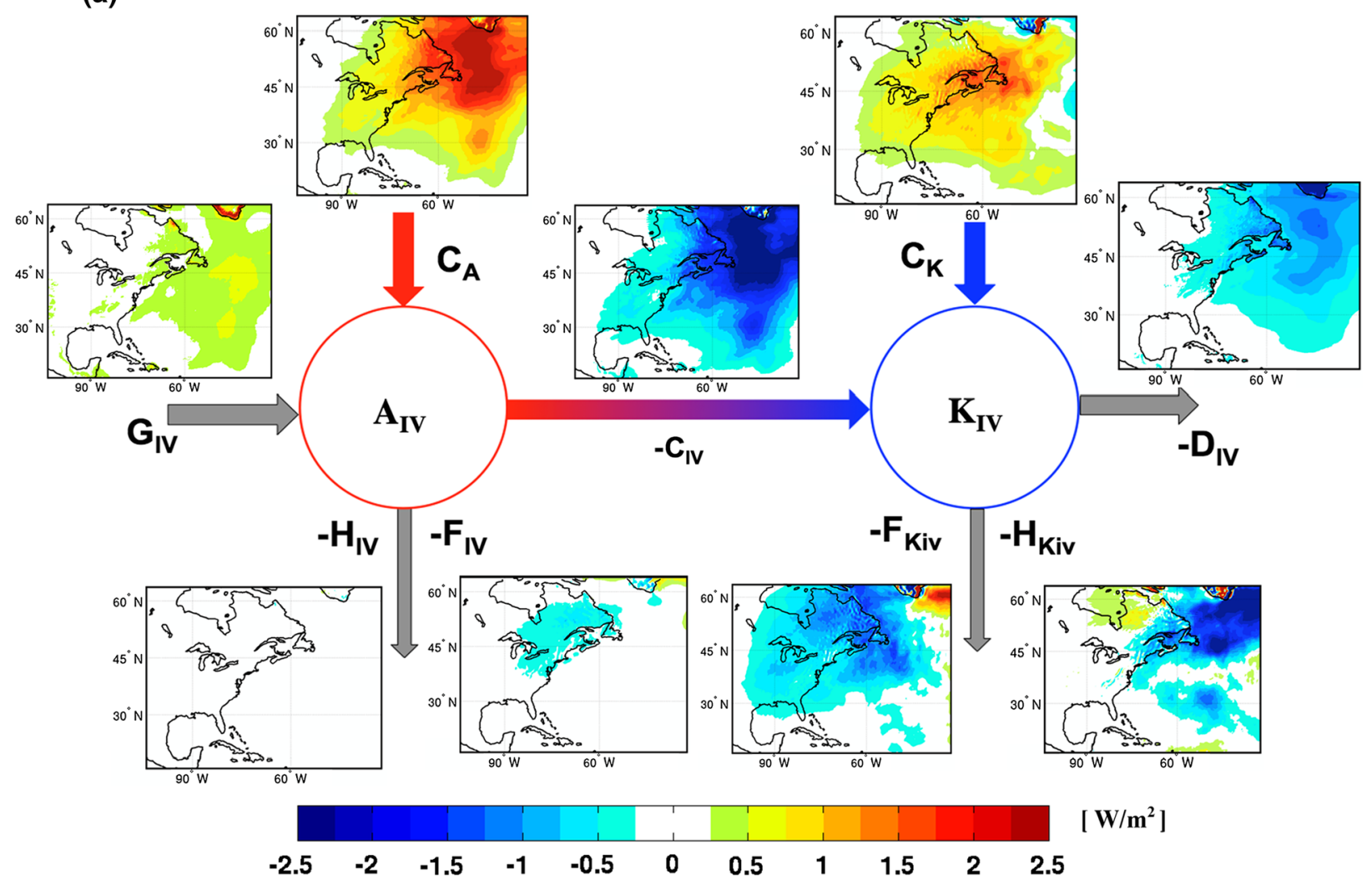

(b)

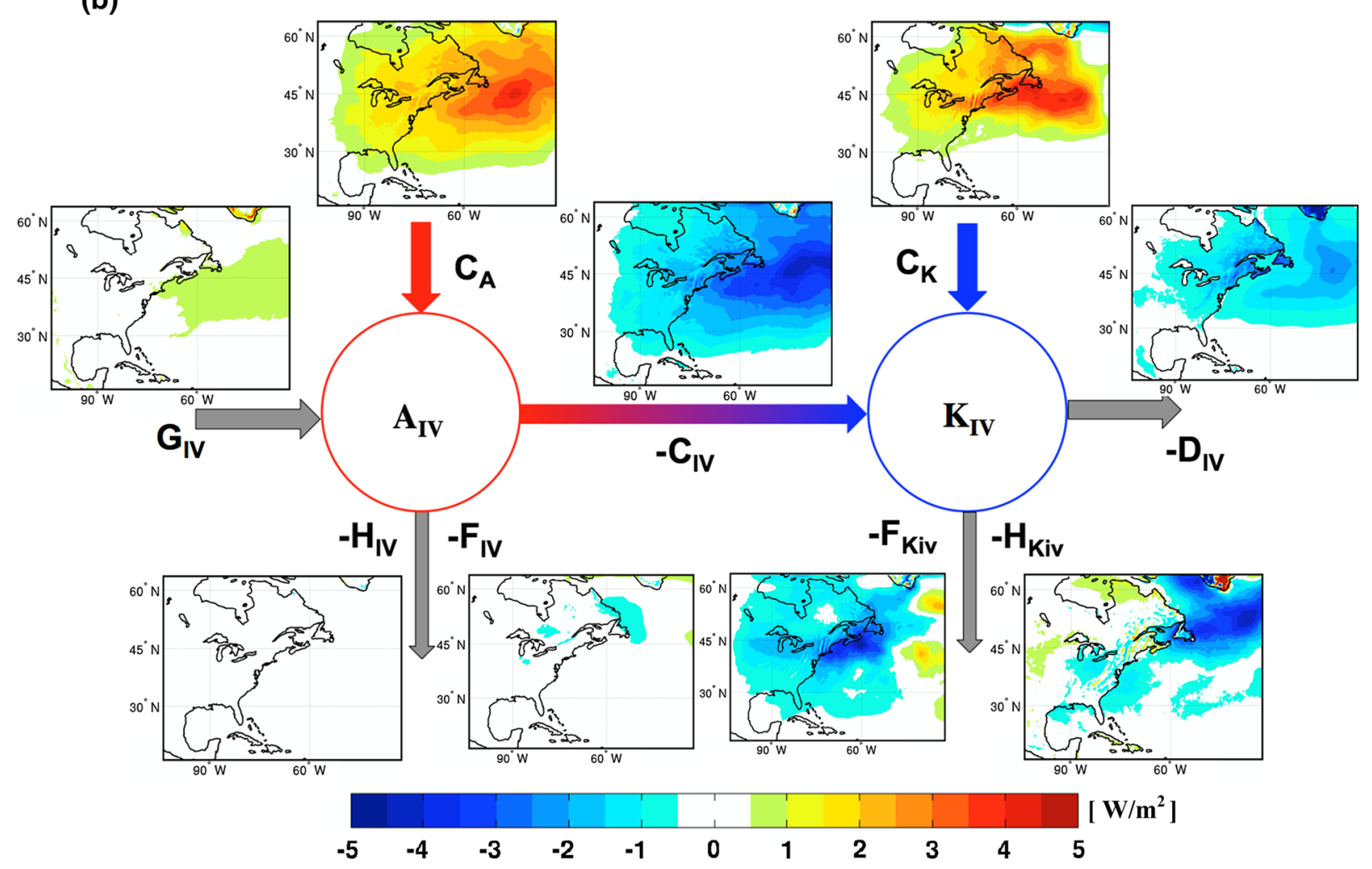

Fig. 6 Maps of seasonal-mean vertically integrated contributions to the budgets of $\mathrm{A}_{\mathrm{IV}}$ and $\mathrm{K}_{\mathrm{IV}}$, for $\mathbf{a}$ winter, $\mathbf{b}$ spring, $\mathbf{c}$ summer and $\mathbf{d}$ autumn 
(c)
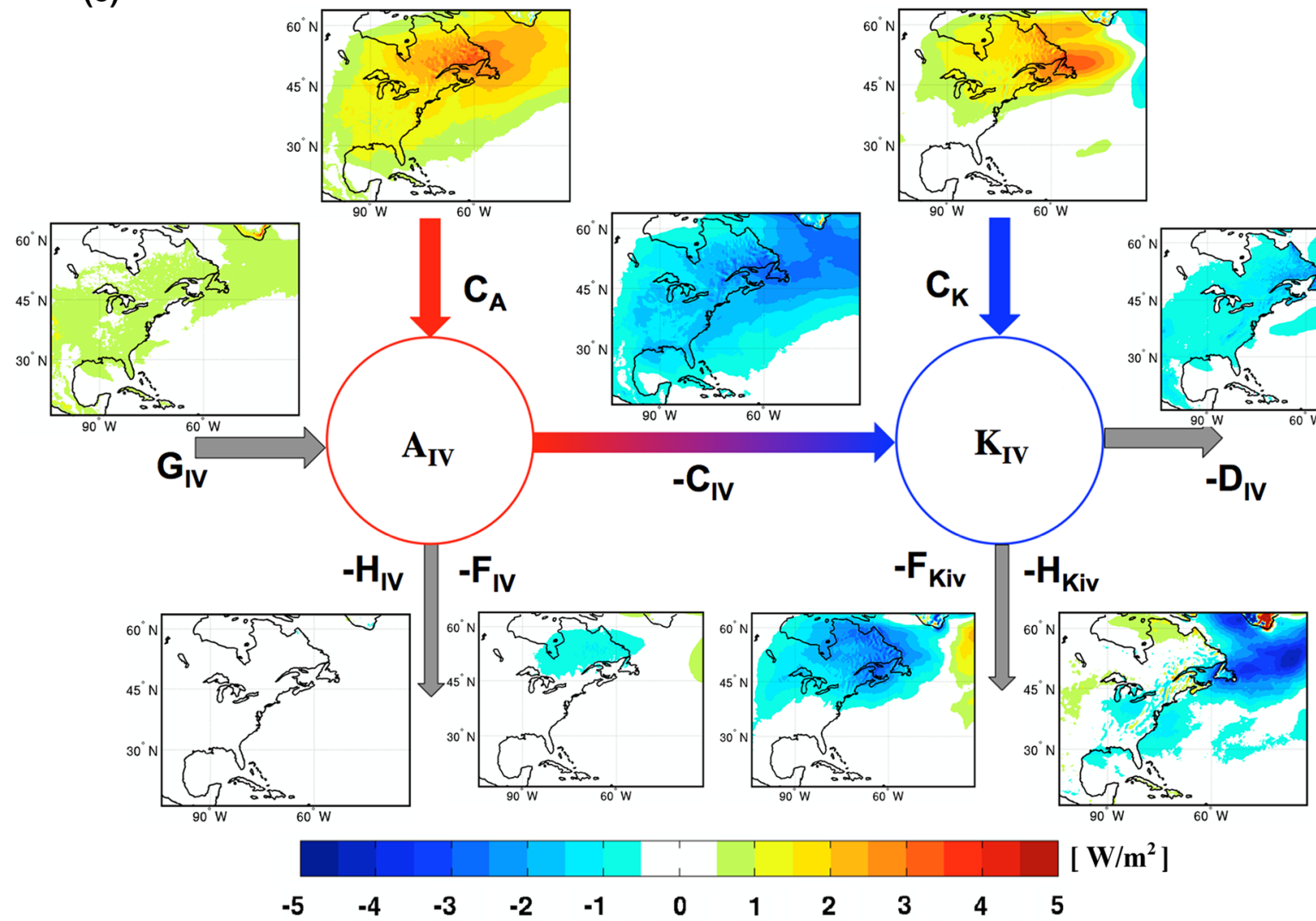

(d)

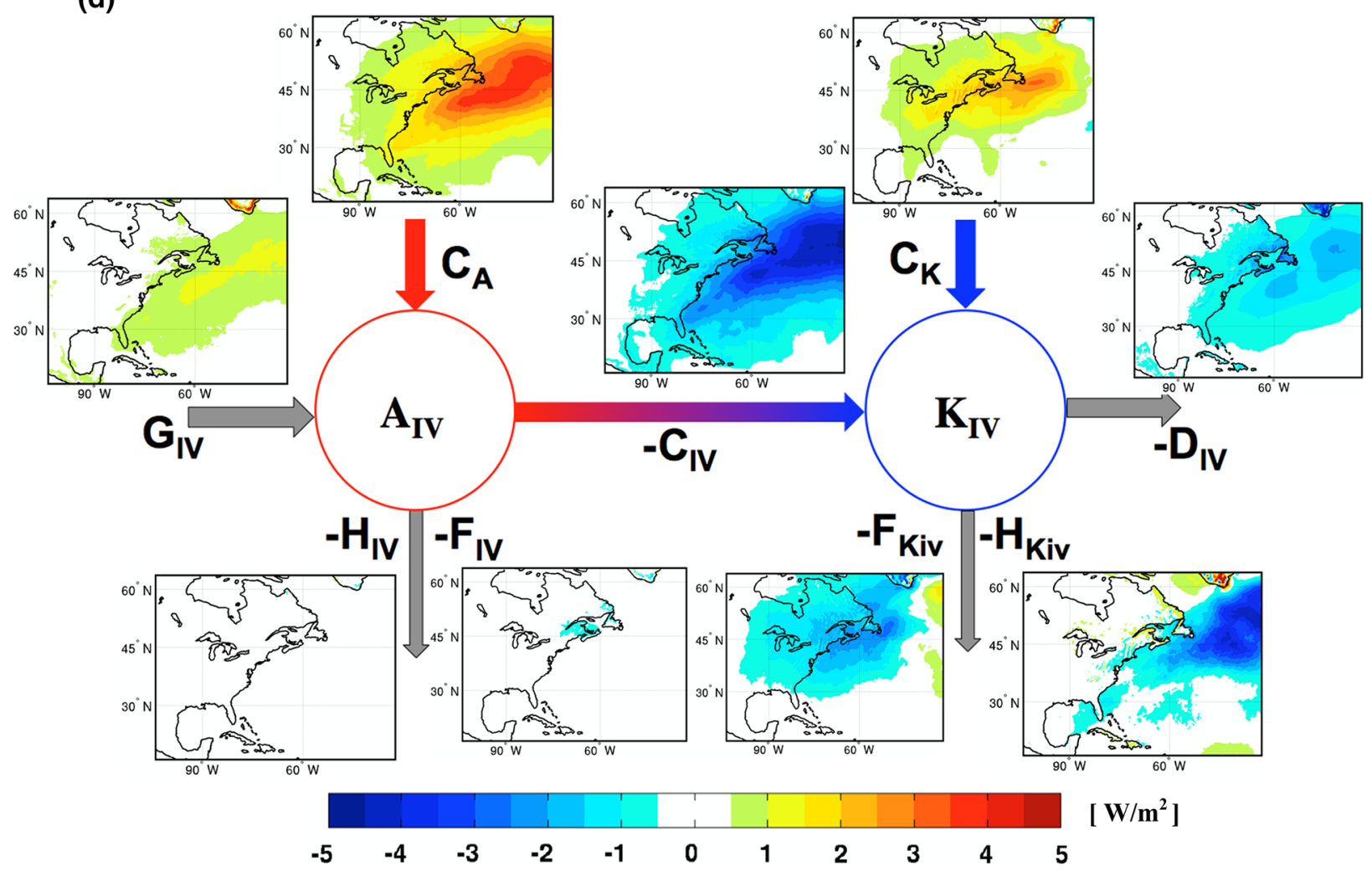

Fig. 6 continued 
(a) $\mathrm{G}_{\mathrm{IV}}$ for 2005-winter

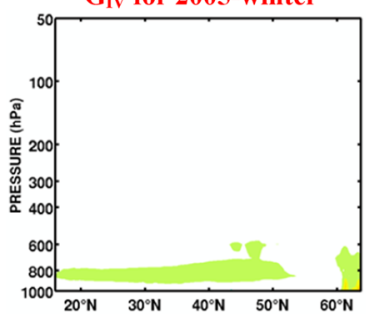

$\mathrm{G}_{\mathrm{TV}}$ for 2005-spring

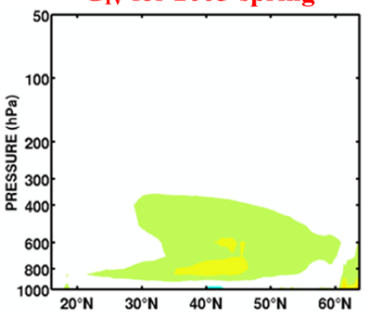

$\mathrm{G}_{\mathrm{IV}}$ for 2005-summer
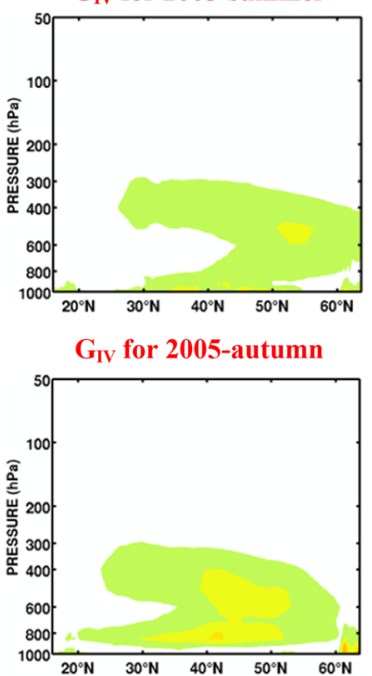
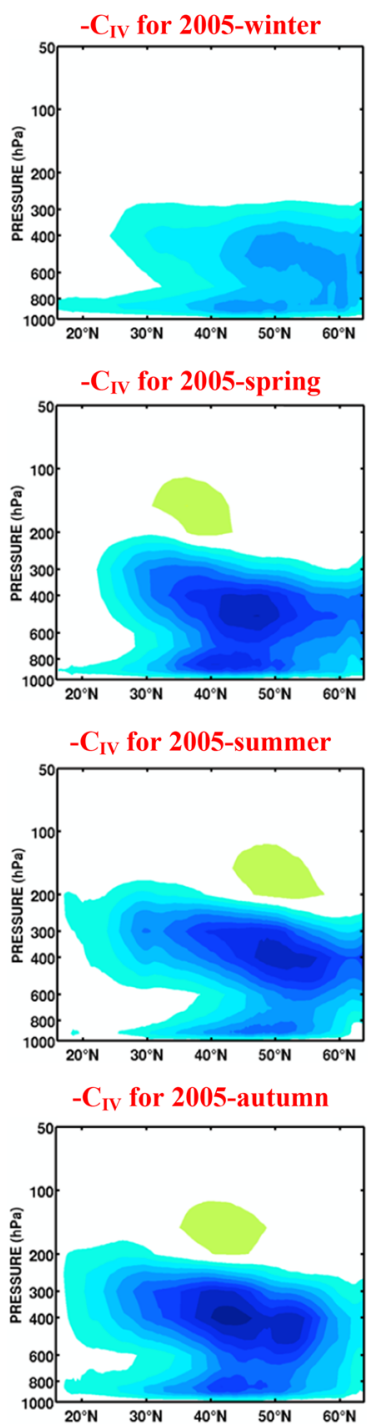
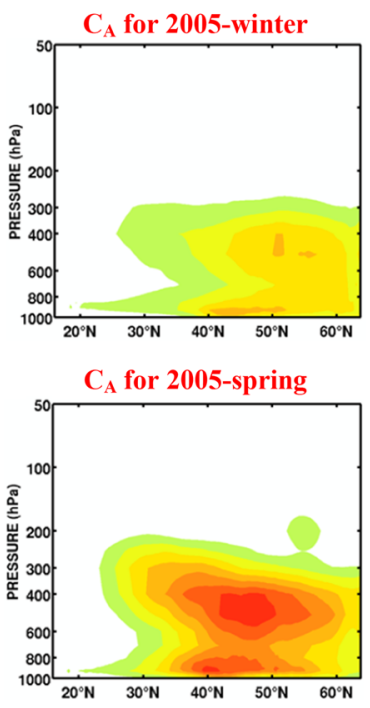

$\mathrm{C}_{\mathrm{A}}$ for 2005-summer

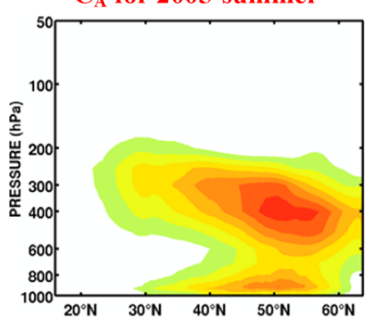

$C_{A}$ for 2005-autumn

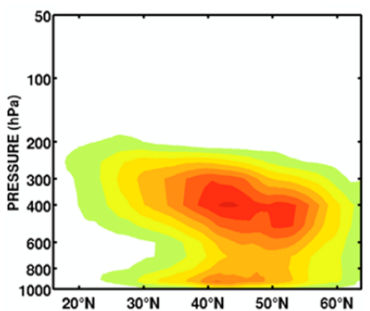

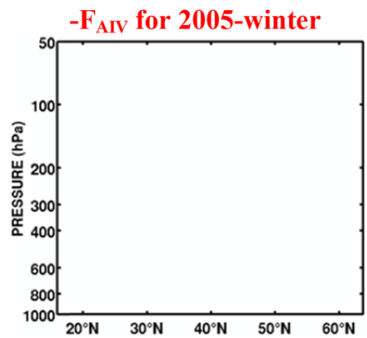

$\left[10^{-4} \mathbf{W} / \mathbf{k g}\right]$
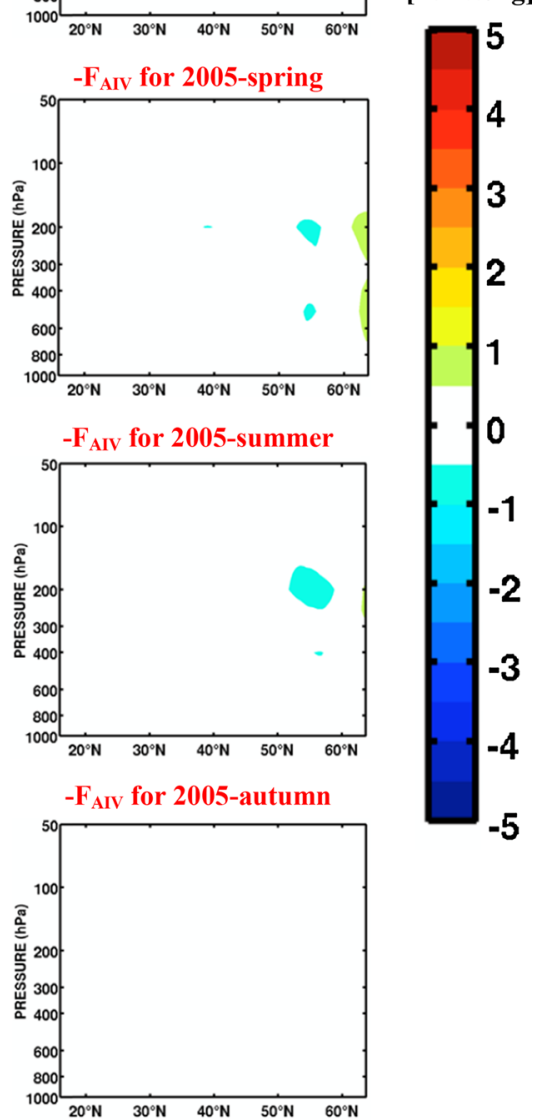

Fig. 7 Vertical cross-section of the longitudinal mean of different contributions to a IV available enthalpy $\left(\mathrm{A}_{\mathrm{IV}}\right)$ budget and $\mathbf{b}$ IV kinetic energy $\left(K_{I V}\right)$ budget. a Terms in the $\mathrm{A}_{\mathrm{IV}}$ budget equation. $\left(-H_{A I V}\right)$ is not shown because of is very weak. $\mathbf{b}$ Terms in the $\mathrm{K}_{\mathrm{IV}}$ budget equation. $\mathrm{C}_{\mathrm{IV}}$ is shown in figure (a)
In the two following sub-sections, we will successively look at the horizontal and vertical structures and time evolution of the various contributions in order to shed some light on the physical meanings of the various energy sources/sinks and exchanges between reservoirs.

\subsubsection{Energy exchanges between inter-member variability reservoirs}

Figure 6 presents the maps of the contributions of the various exchange terms in the IV available enthalpy $\left(A_{I V}\right)$ and IV kinetic energy $\left(K_{I V}\right)$ budget equations, for each season. We note that, in a vertically integrated and timeaveraged sense, most individual contributions tend to act systematically as either positive or negative contribution to the IV energies.

Figure 7 shows meridional-vertical cross-sections of the longitudinal mean of various contributions to $A_{I V}$ and $K_{I V}$ budget equations. At least in the troposphere, panels in Fig. 7 confirm the systematic positive or negative contributions of each term, except for the term $\left(-H_{K I V}\right)$ that exhibits negative sign in mid-troposphere and positive sign near the surface and above the tropopause; this result will be commented upon later in sub-Sect. 3.4.

The conversion terms $C_{A}$ and $C_{K}$ are positive in all seasons, which corresponds to transfers of energy from EM to IV reservoirs. The term $C_{A}$ corresponds to covariance of temperature and flow anomalies acting upon the 

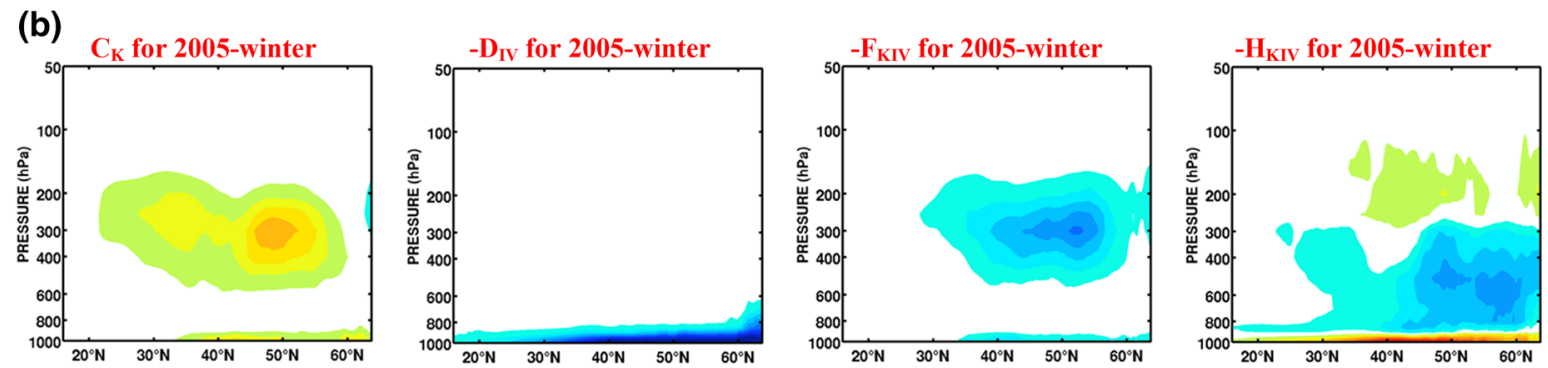

$\left[10^{-4} \mathrm{~W} / \mathrm{kg}\right]$
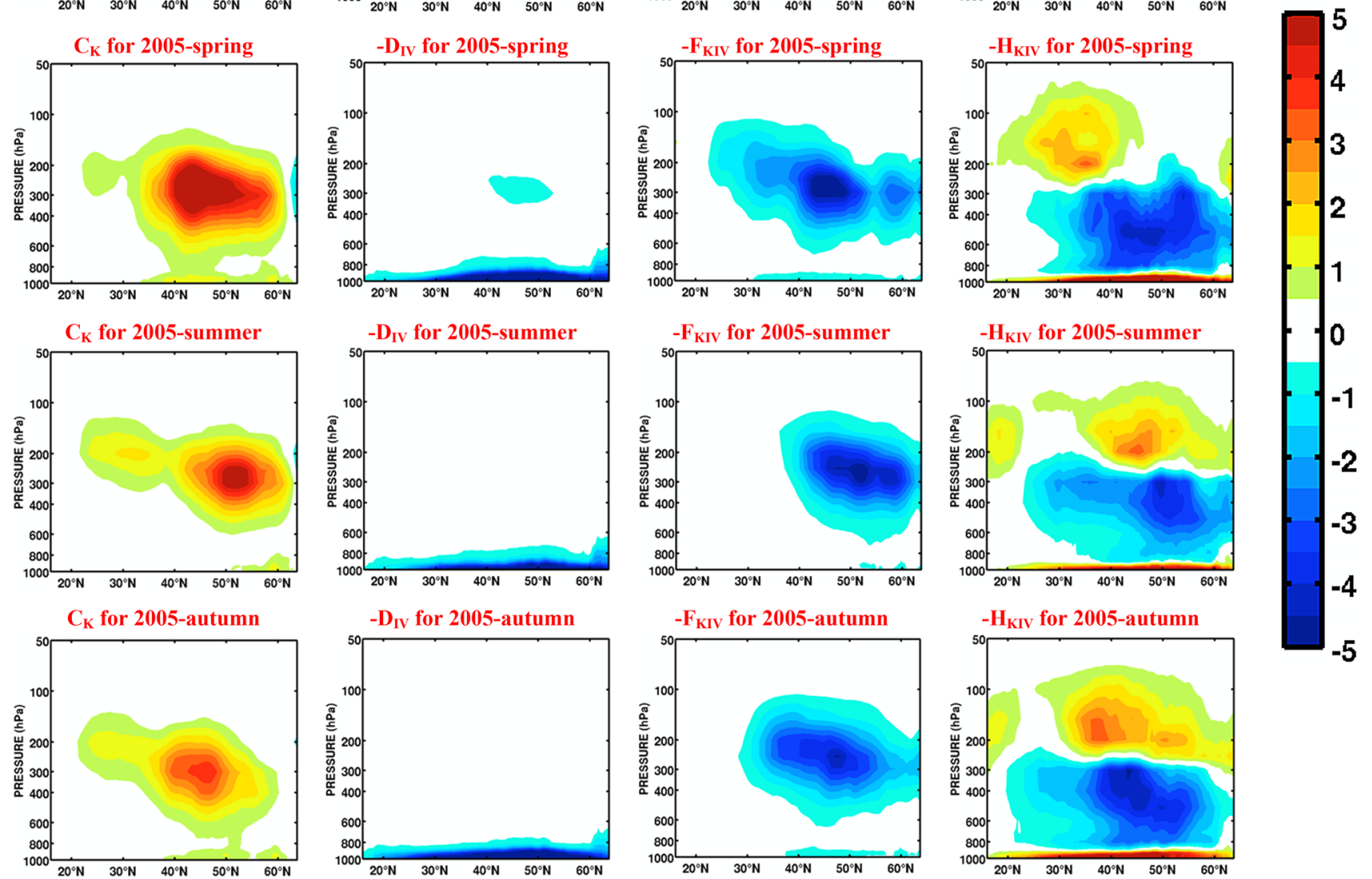

Fig. 7 continued

Fig. 8 Time evolutions of the conversion term $\mathrm{C}_{\mathrm{A}}$ with its horizontal $\left(\mathrm{C}_{\mathrm{AH}}\right)$ and vertical $\left(\mathrm{C}_{\mathrm{AV}}\right)$ components

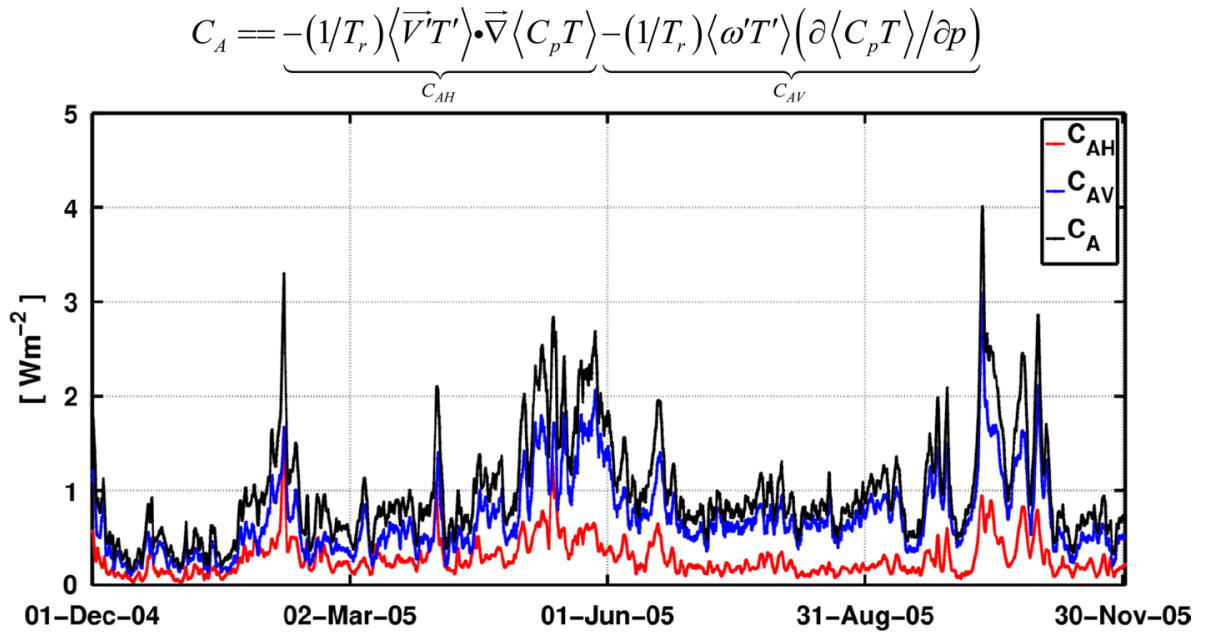


EM temperature gradient, and the term $C_{K}$ corresponds to covariance of components of flow anomalies acting upon the EM flow gradient. The fact that these terms are positive implies that the eddy fluxes are down the gradient of the EM state. As mentioned at the end of Sect. 3.2, IV energies are important in spring and autumn, and in summer (see Fig. 4), because of the conversion terms that feed mainly IV reservoirs (see Figs. 1, 6). In spring and autumn, the terms $C_{A}$ and $C_{K}$ are important compared to their values in summer due to higher values of covariances of perturbations $\left(\left\langle\overrightarrow{V^{\prime}} T^{\prime}\right\rangle,\left\langle\omega^{\prime} T^{\prime}\right\rangle,\left\langle\overrightarrow{V^{\prime}} \vec{V}^{\prime}\right\rangle\right.$ and $\left\langle\omega^{\prime} \vec{V}^{\prime}\right\rangle$, result not shown) that are down the gradient of the ensemble-means states $(\vec{\nabla}\langle T\rangle, \partial\langle T\rangle / \partial p, \vec{\nabla}\langle\vec{V}\rangle$ and $\partial\langle\vec{V}\rangle / \partial p)$.

The term $C_{I V}$ represents a conversion between $A_{I V}$ and $K_{I V}$. Figures 6 and $7 \mathrm{a}$ show that $-C_{I V}=\left\langle\omega^{\prime} \alpha^{\prime}\right\rangle\langle 0$ in all seasons, so this term acts systematically to transfers energy from $A_{I V}$ to $K_{I V}$. This reflects the fact that anomalies of vertical motion $\omega$ and temperature are negatively correlated, $\left\langle\omega^{\prime} T^{\prime}\right\rangle<0$, meaning that vertically upward (downward) motion anomalies accompany warm (cold) anomalies, on average. This is equivalent to baroclinic conversion from perturbation potential energy to perturbation kinetic energy in weather systems (e.g., Lorenz 1955, 1967).

The contributions of diabatic processes (such as condensation, convection and radiation) are contained in the term $G_{I V}=l\left\langle T^{\prime} Q^{\prime} / T_{r}\right\rangle$. Figures 6 and 7 a show that this term acts as a source for $A_{I V}$. This implies that diabatic heating anomalies and temperature anomalies in the ensemble are positively correlated, as noted before by Nikiéma and Laprise (2011a, b).

Figures 6 and $7 \mathrm{~b}$ also indicate that in all seasons $K_{I V}$ is lost through dissipation processes in term $-D_{I V}=\left\langle\overrightarrow{V^{\prime}} \cdot \overrightarrow{F^{\prime}}\right\rangle$, in a similar fashion to weather systems.

As shown in Figs. 5 and 7, the terms $F_{A I V}$ and $F_{K I V}$ act systematically as large sinks for $A_{I V}$ and $K_{I V}$, respectively; this represents the fact that $A_{I V}$ and $K_{I V}$ are transported out of the regional domain by the EM flow. Figure $7 \mathrm{~b}$ reveals that the vertical cross-section of $\left(-F_{K I V}\right)$ acts upon $K_{I V}$ mostly in the high-levels of the troposphere (see thirdcolumn panels), with maximum contribution in the vicinity of the tropopause $(250 \mathrm{hPa})$. This result confirms that the faster upper-tropospheric winds near jet-stream level favour the transport of IV kinetic energy out of the regional domain, thus contributing to reduce the IV kinetic energy.

To summarise, our results reveal that the $A_{I V}$ reservoir is fed by the term $C_{A}$ at the expense of the $A_{E M}$ reservoir, with the diabatic term $G_{I V}$ also contributing to fill the $A_{I V}$ reservoir but to a lesser extent. The reservoir $A_{I V}$ is mostly converted to $K_{I V}$ through the "baroclinic" term $C_{I V}$. The $K_{I V}$ reservoir is also fed by the $C_{K}$ term that converts energy from $K_{E M}$. Finally the $K_{I V}$ reservoir is drained by dissipation in the $D_{I V}$ term and advection out of the domain by the (a)

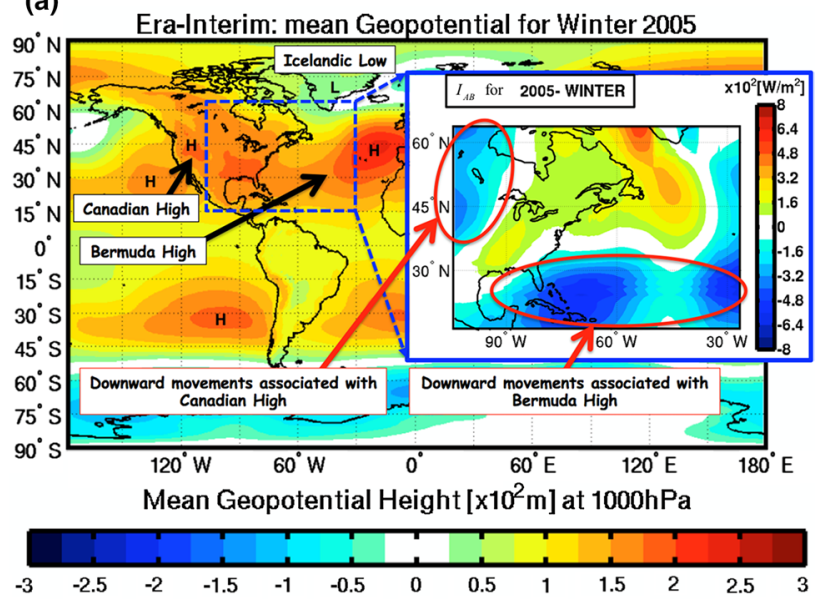

(b)

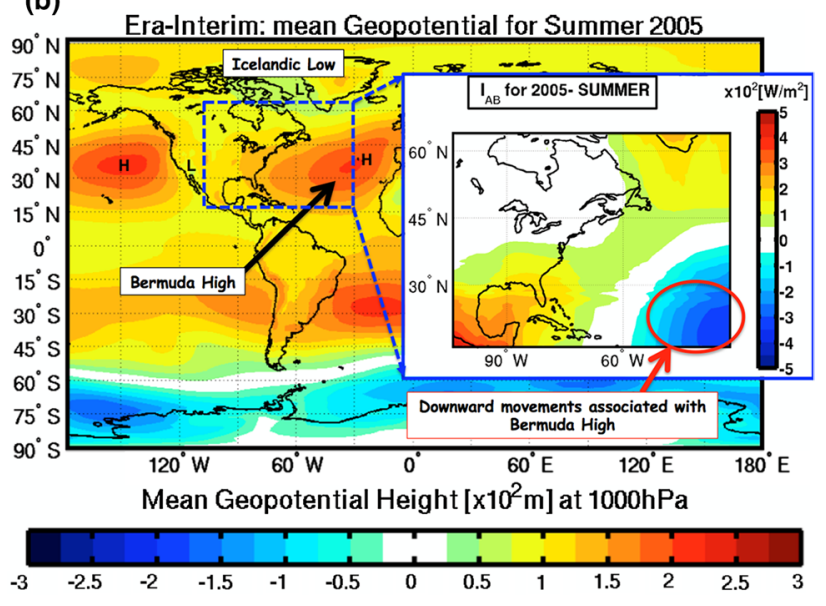

Fig. 9 2005-Seasonal (winter and summer) maps of geopotential height from Era-Interim data and the seasonal maps of the term $\mathrm{I}_{\mathrm{AB}}$ in the $\mathrm{A}_{\mathrm{EM}}$ budget equation

$F_{K I V}$ term. Note that, at the seasonal scale, the third-order term $H_{A I V}$ is negligible in the $A_{I V}$ budget, while the term $H_{K I V}$ acts as a weak sink in the $K_{I V}$ budget.

To gain some further physical insight, the term $C_{A}$ is split into its two components, $C_{A H}=-\left(1 / T_{r}\right)\left\langle\vec{V}^{\prime} T^{\prime}\right\rangle$. $\vec{\nabla}\left\langle C_{p} T\right\rangle$ and $C_{A V}=-\left(1 / T_{r}\right)\left\langle\omega^{\prime} T^{\prime}\right\rangle \frac{\partial\left\langle C_{p} T\right\rangle}{\partial p}$, and the time evolution of their domain-averaged values is displayed in Fig. 8. Both contributions are positive at all time, which implies transfer energy from the $A_{E M}$ reservoir to the $A_{I V}$ reservoir, as mentioned before. The larger component $C_{A V}$ is positive because $\left\langle\omega^{\prime} T^{\prime}\right\rangle$ is negative and $\partial\langle T\rangle / \partial p$ generally positive over most of the troposphere. Physically, a negative covariance means that, on average, warm (cold) anomalies have an upward (downward) motion anomaly, thus transporting heat down the gradient. The smaller component $C_{A H}$ is also positive sign, which implies that the covariance of temperature and horizontal flow anomaly 
Fig. 10 Regional energy cycle on pressure surfaces associated with inter-member variability (IV) in ensemble of RCM simulations [reproduced with modifications from Nikiéma and Laprise (2013) (C) 2013 Springer-see Fig. 2]. The values represent the seasonal mean of different terms integrated over the whole regional domain

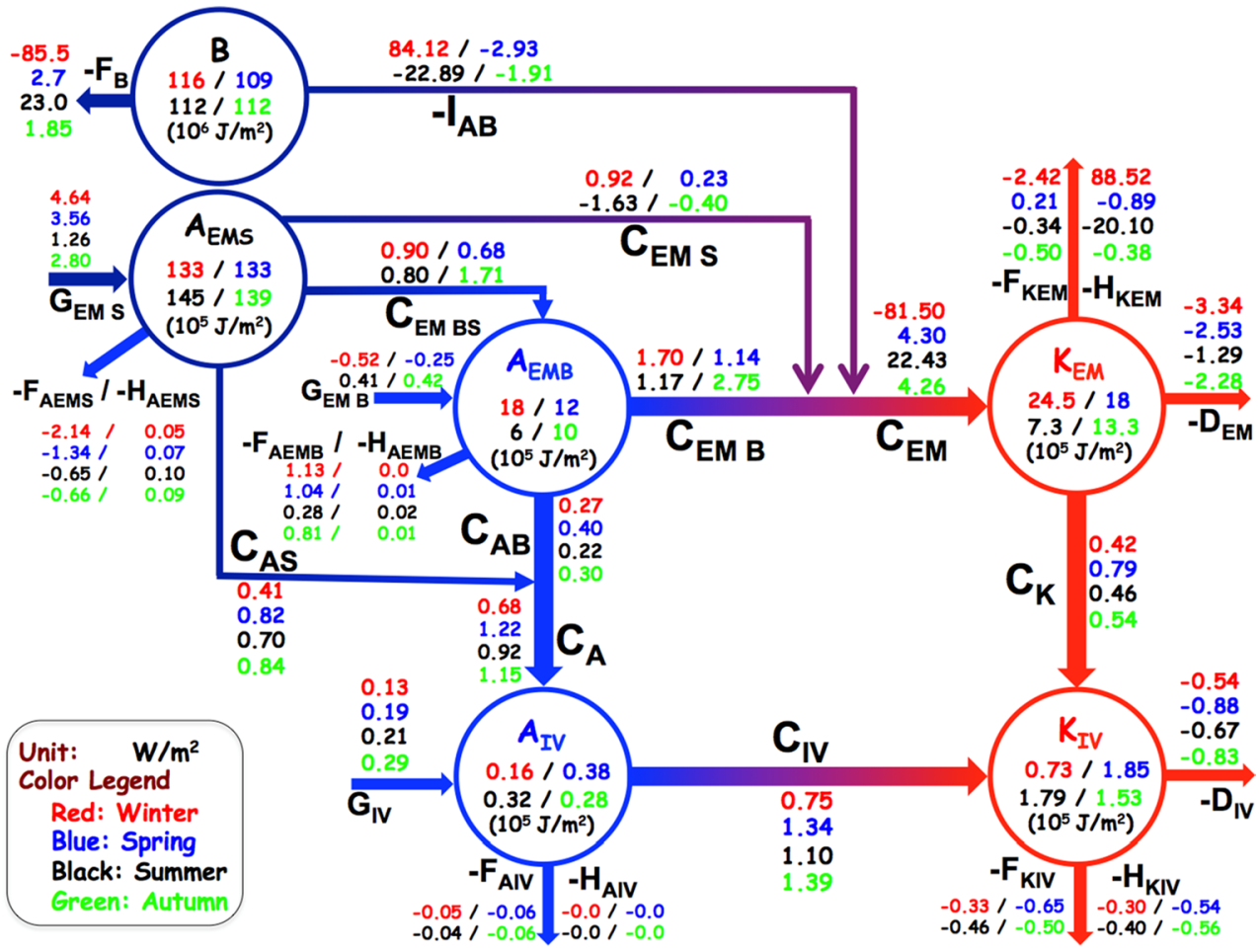

vector are directed against the EM temperature gradient. In the meridional direction, this means that $\left\langle v^{\prime} T^{\prime}\right\rangle>0$ on average. Physically, this means that anomalies transport warm air poleward and cold air equatorward.

\subsubsection{Energy exchanges between ensemble-mean reservoirs}

Figure $5 \mathrm{c}, \mathrm{d}$ showed the different contributions to EM available enthalpy $\left(A_{E M}\right)$ and EM kinetic energy $\left(K_{E M}\right)$ reservoirs, for the four seasons in 2005. On average, $G_{E M}$ and $I_{A B}$ act as energy sources for $A_{E M}$, except in winter where $I_{A B}$ is a large negative contribution $\left(\sim-84 \mathrm{~W} / \mathrm{m}^{2}\right.$, see Fig. 1). Since the reference temperature and pressure have positive signs, the negative sign of $I_{A B}(=-(R T, / p)\langle\omega\rangle)$ is associated with a positive vertical motion $(\langle\omega\rangle>0$ i.e. downward motion), on average in the study domain (result not shown). The strong negative contribution of $I_{A B}$ over the Caribbean corresponds to the subsiding branch of the Hadley circulation in the subtropics. Figure 9a shows the mean sea level pressure map in winter. Over the region of interest, there are the two large persistent surface atmospheric high-pressure centres, namely the Canadian High located over the continental North America and the Bermuda High located over the Azores extending westward to Bermuda over the North Atlantic Ocean, and one low-pressure centre, the Icelandic Low extending westward to the Labrador Sea. The insert in Fig. 9a shows a good correspondence between the negative contribution of $I_{A B}$ and the expected subsidence associated with the high-pressure centres and the Hadley circulation. In summer the Hadley circulation pattern moves southward, and hence subsidence over the Caribbean weakens. Figure 9b shows that the Atlantic high pressure moves westward towards Bermuda. The pattern of $I_{A B}$ reflects overall these changes.

For the $A_{E M}$ reservoir, the terms $C_{E M}$ and $I_{A B}$ act in opposite way with the same order of magnitude (Figs. 1, 5c) because the EM temperature $(\langle T\rangle)$ only deviates from the reference temperature $\operatorname{Tr}\left(=260^{\circ} \mathrm{K}\right)$ by $<5 \%$ (result not shown). $C_{E M}$ is the conversion term between the two EM energies $\left(A_{E M}\right.$ and $\left.K_{E M}\right)$. For $K_{E M}$ budget, the large value of $C_{E M}$ in winter is mainly offset by $H_{K E M}$ due to the large values of the horizontal divergence of EM geopotential flux [see the equation for $H_{K E M}$ in Eq. (8)]. Results also confirm that the EM kinetic energy is lost by the dissipation processes in the term $D_{E M}$ (see Figs. 1, 5d).

\subsection{Decomposition on isobaric surface and deviation thereof}

Figure 10 presents the regional energy cycle as in Fig. 1, but with the $A_{E M}$ reservoir further decomposed into mean stratification $\left(A_{E M S}\right)$ and baroclinicity $\left(A_{E M B}\right)$. As mentioned previously, the baroclinic term $\left(A_{E M B}\right)$ has the same order as $K_{E M}$. In parallel with energy conversion in weather system, $A_{E M B}$ can be attributed to available energy, i.e. potential energy that is available to be converted into kinetic energy, while $A_{E M S}$ is unavailable. The $A_{E M B}$ reservoir is fed 
Fig. 11 Vertical profiles of different terms in $\mathrm{A}_{\mathrm{IV}}, \mathrm{K}_{\mathrm{IV}}, \mathrm{A}_{\mathrm{EMB}}$ and $\mathrm{K}_{\mathrm{EM}}$ budget equations on pressure surface. a $\mathrm{A}_{\mathrm{IV}}$ budget on isobaric surface. $\mathbf{b} \mathrm{K}_{\mathrm{IV}}$ budget on isobaric surface. c $\mathrm{A}_{\mathrm{EMB}}$ budget on isobaric surface. $\mathbf{d ~} \mathrm{K}_{\mathrm{EM}}$ budget on isobaric surface

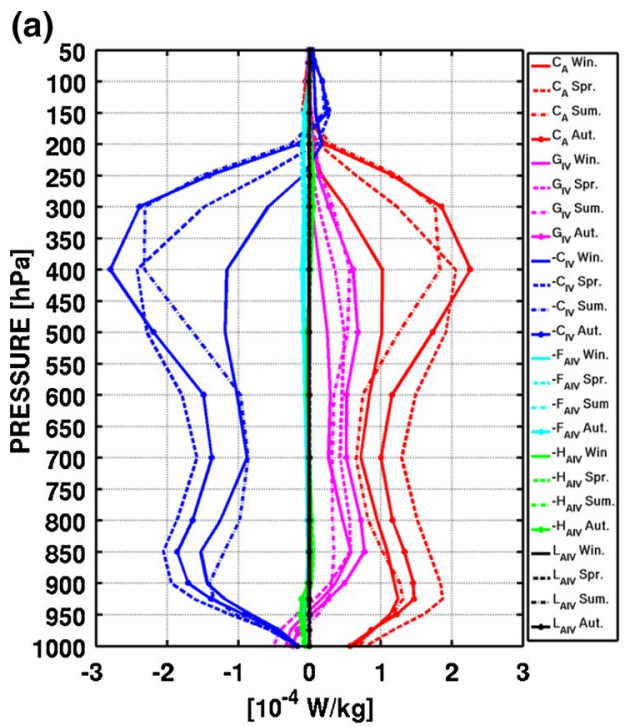

(c)

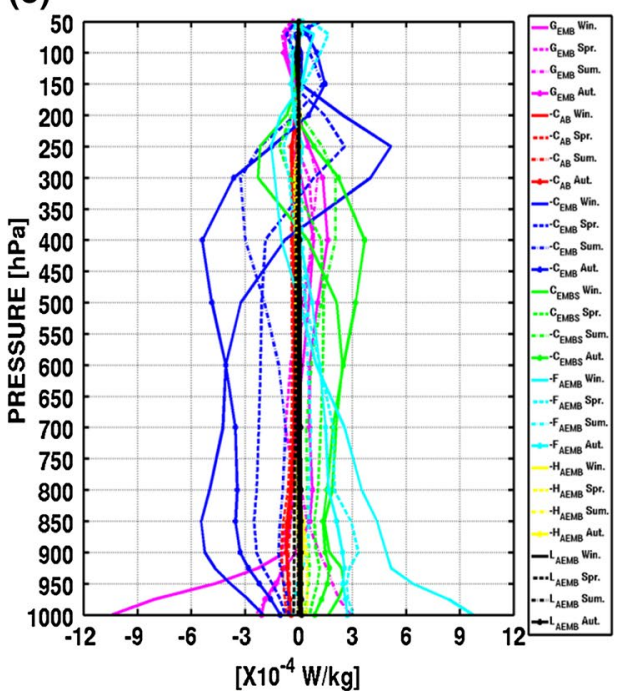

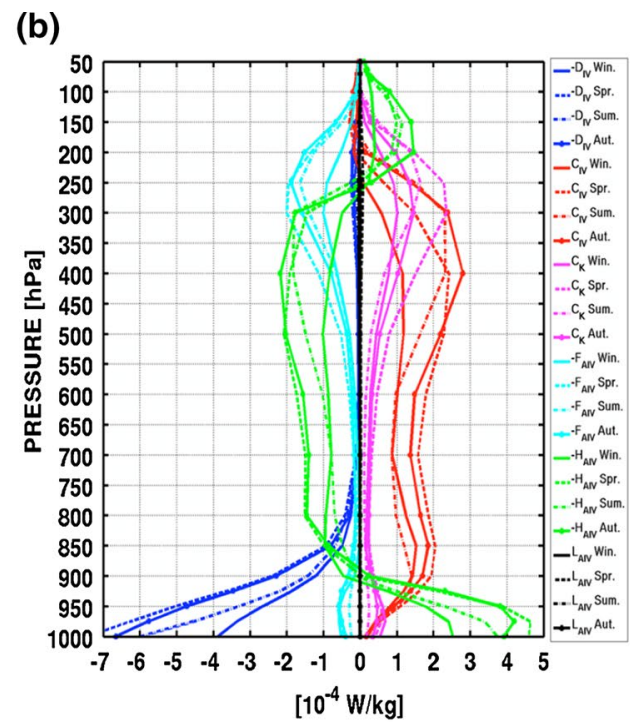

(b)

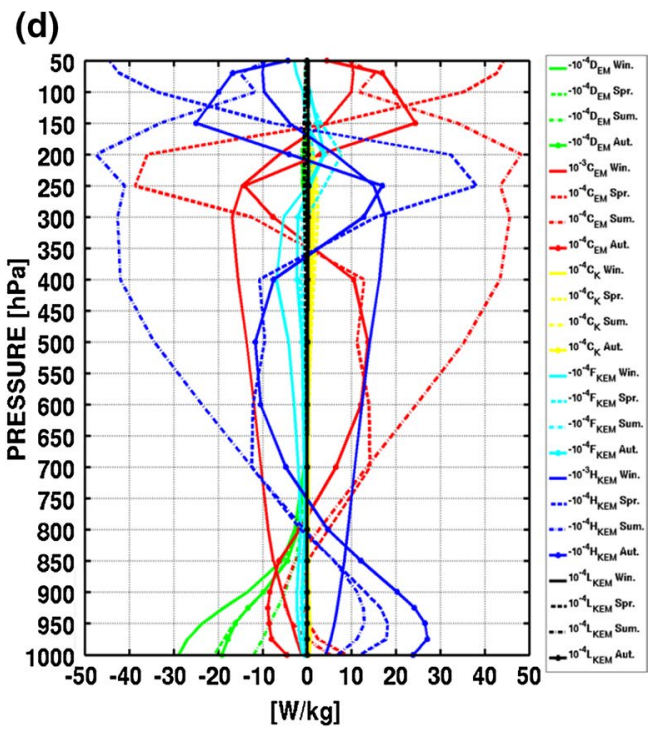

by $A_{E M S}$ via the conversion term $C_{E M B S}$. On average, this conversion term has a positive sign at all pressure levels in the troposphere for all seasons (see green lines in Fig. 11c). Contrary to what has been seen for the others reservoirs, the transport term $-F_{A E M B}$ of $A_{E M B}$ contributes positively at all seasons (see Fig. 10), mainly in the lower troposphere (see blue light plots in Fig. 11c). $C_{E M B}$ and $C_{A B}$ are conversion terms from $A_{E M B}$ to $K_{E M}$ and to $A_{I V}$, respectively; they are parts of the large conversion terms $C_{E M}$ and $C_{A}$, respectively. The EM available enthalpy due to stratification $\left(A_{E M S}\right)$ is fed by the term $G_{E M S}$ due to processes associated with condensation, convection and radiation (result not shown). $A_{E M S}$ is lost by transport out of the regional domain in the term $-F_{A E M S}$ and by conversion in the terms $C_{E M S}$ and $C_{A S}$.

Figure 11 shows the vertical profiles of various terms in the $A_{I V}, K_{I V}, A_{E M B}$ and $K_{E M}$ budgets. For all reservoirs, results reveal that the tendency terms $\left(L_{E}\right)$ nearly vanish at all levels as a result of an approximate balance between positive and negative contributions. This means that there is very little energy trend at the seasonal scale. For $A_{I V}$, it is clearly shown that the positive contributions, namely $G_{I V}$ and $C_{A}$, are counterbalanced by $C_{I V}$ at all levels (see Fig. 11a). Figure 11b shows the contributions for the $K_{I V}$ budget where the term $C_{K}$ is balanced by the transport term $-F_{K I V}$ at all levels, with maximum values near the tropopause, whereas $C_{I V}$ is offset by $-H_{K I V}$ in the mid-troposphere. But near the surface, $-H_{K I V}$ is offset by the dissipation term $-D_{I V}$. For $K_{E M}$, Fig. $11 \mathrm{~d}$ shows the same result where $-H_{K E M}$ is offset by $-D_{E M}$ near the surface.

The time evolution of horizontal and vertical gradients of $H_{K I V},\left(\vec{\nabla} \cdot\left\langle\left(k+\Phi^{\prime}\right) \vec{V}^{\prime}\right\rangle\right)$ and $\left(\partial\left\langle\left(\Phi^{\prime}+k\right) \omega^{\prime}\right\rangle / \partial p\right.$, respectively, with $k=\overrightarrow{V^{\prime}} \cdot \overrightarrow{V^{\prime}} / 2$ ), indicates a positive sign 
Fig. 12 a Time evolution of $\mathrm{H}_{\mathrm{KIV}}$ with its components due to horizontal and vertical gradients. Vertical profiles of $\mathbf{b}$ horizontal and vertical gradients of $-\mathrm{H}_{\mathrm{KIV}}$, and $\mathbf{c}$ the covariance of fluctuations of geopotential height and vertical motion for 2005-seasons

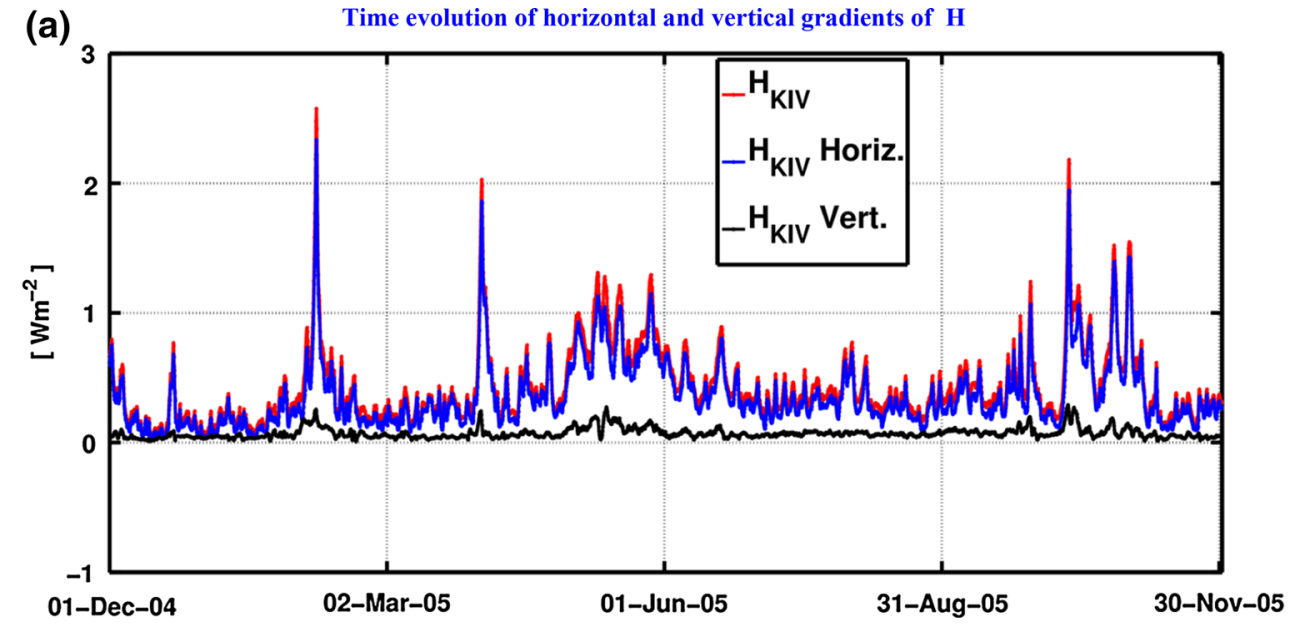

(b) Horizontal and vertical gradients of $-\mathrm{H}_{\mathrm{KIV}}$

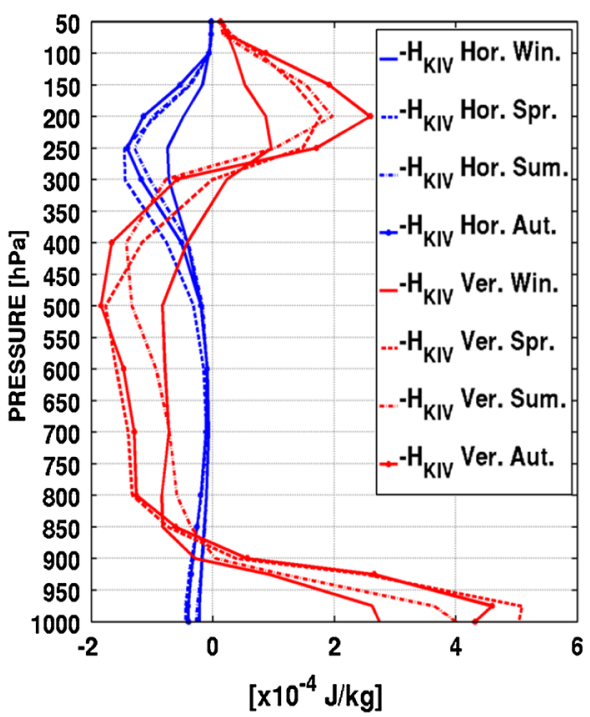

(c) Covariance of fluctuations of geopotential height and

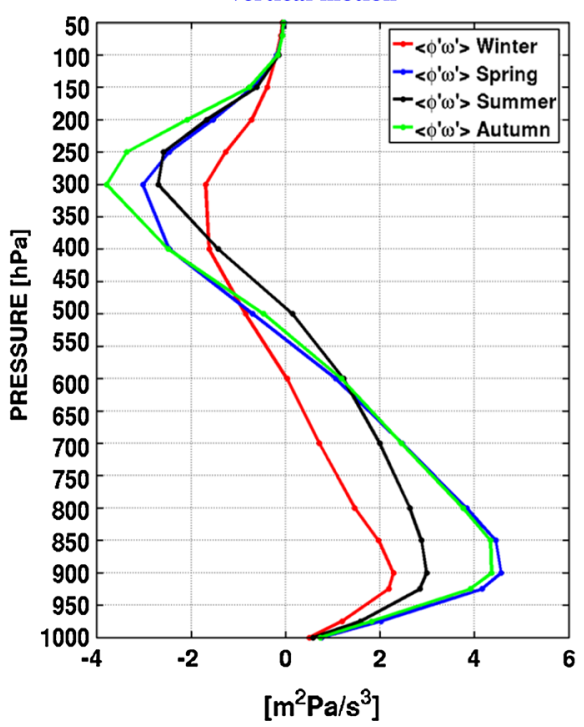

for both, mostly due to a large contribution of the horizontal gradient term (see Fig. 12a), resulting in a negative contribution on average of $-H_{K I V}$ to $K_{I V}$ budget (as mentioned previously; see Figs. 1, 5b). Figure 12b compared to Fig. $11 \mathrm{~b}$ reveals that the vertical distribution of $-H_{K I V}$ is due to its vertical gradient term $\left(-\partial\left\langle\left(\Phi^{\prime}+k\right) \omega^{\prime}\right\rangle / \partial p\right)$. Since $-\partial\left\langle k \omega^{\prime}\right\rangle / \partial p$ is a small third-order term (not shown), these results reveal that the vertical gradient of the covariance of vertical motion and geopotential fluctuations $\left(-\partial\left\langle\omega^{\prime} \Phi^{\prime}\right\rangle / \partial p\right)$ is linked to the friction processes $\left(\left\langle\overrightarrow{V^{\prime}} \cdot \overrightarrow{F^{\prime}}\right\rangle\right)$, in agreement with Ekman pumping mechanism. Near the ground, $\partial\left\langle\omega^{\prime} \Phi^{\prime}\right\rangle / \partial p$ is a negative gradient (i.e. $\left\langle\omega^{\prime} \Phi^{\prime}\right\rangle$ increases with height (see Fig. 12c), indicating that negative geopotential perturbations $\left(\Phi^{\prime}<0\right)$ are associated with upward vertical motion perturbations $\left(\omega^{\prime}<0\right)$. Above the $900 \mathrm{hPa}$ level in the troposphere, $-H_{K I V}$ acts negatively to $K_{I V}$ tendency due to positive vertical gradients of $\left\langle\omega^{\prime} \Phi^{\prime}\right\rangle$ (see Fig. 12c).

\section{Summary and conclusion}

Following the paper of NL13 where an approximate formulation was proposed for the energy cycle of IV, this study is done to document the IV energy cycle by using a large ensemble of RCM simulations driven by identical boundary conditions. In a 50-member ensemble whose members differ only in their initial conditions, the various energy reservoirs of IV and exchange terms between reservoirs were evaluated in 1-year (2005) simulations performed with the fifth-generation Canadian RCM (CRCM5) over an eastern North American domain. Results were analysed and some physical interpretations were made.

Results showed a remarkably close parallel between the energy conversions associated with IV in ensemble simulations and the energy conversions in weather systems. Indeed, the energy conversions associated with IV perturbations appear to behave in a similar fashion to those of 
transient-eddy energy (e.g., Lorenz 1955, 1967; Pearce 1978; Marquet 2003a, b). The fluctuations of available enthalpy $\left(A_{I V}\right)$ are generated by diabatic processes such as condensation, convection and radiation (term $G$ ), and this energy is converted to IV kinetic energy $\left(K_{I V}\right)$ trough the conversion term $C_{I V}$. Results also show that kinetic energy due to IV is destroyed by dissipation processes (term $D$ ). Because the simulations were performed over a limit-area domain, some boundary flux terms ( $F$ and $H$ ) also contribute to IV energy budgets; these terms would not have any contribution over the whole globe. Physically, the terms $F$ contribute to reduce IV energies by their transport out of the regional domain.

This study reveals that RCM's IV is a natural phenomenon arising from the chaotic nature of the atmosphere. In term of perspective, similar study can be done to analyze seasonal transient-eddy energy in comparison with those obtain in the present study. Since it is known that the RCM's IV is smaller than the transient eddy variability, such studies can help to get further understanding on RCM's uncertainties.

Acknowledgments This research was funded by the Québec's Ministère du Développement Économique, Innovation et Exportation (MDEIE), Hydro-Québec, the OURANOS Consortium on Regional Climatology and Adaptation to Climate Change, and the Climate Change and Atmospheric Research (CCAR) programme of the Natural Sciences and Engineering Research Council of Canada (NSERC) through a grant to the Canadian Network for Regional Climate and Weather Processes (CNRCWP). The calculations were made possible through Compute Canada-Calcul Québec supercomputers. The authors thank Mr. Georges Huard and Mrs. Nadjet Labassi and Mrs. Katja Winger for maintaining an efficient and user-friendly local computing facility.

Open Access This article is distributed under the terms of the Creative Commons Attribution 4.0 International License (http://creativecommons.org/licenses/by/4.0/), which permits unrestricted use, distribution, and reproduction in any medium, provided you give appropriate credit to the original author(s) and the source, provide a link to the Creative Commons license, and indicate if changes were made.

\section{Appendix}

We note that the domain integrals of $K_{I V}$ and $A_{I V}$ fluctuate synchronously with a high degree of correlation $(\sim 0.96)$, with $K_{I V} \approx 5 A_{I V}$. This may seem surprising given that the momentum and thermodynamic variables follow distinct equations. We have however to remember that at the scale we are operating, the atmosphere is in hydrostatic and quasi-geostrophic equilibrium. Hence the thermal-wind relationship must be satisfied for the simulated atmosphere, including for the departures from the ensemble-mean state: $\frac{\partial \vec{V}^{\prime}}{\partial \ln p}=-\frac{R}{f} \hat{k} \times \vec{\nabla} T^{\prime}$

For simplicity, let us consider a temperature perturbation $T$ with an equivalent-barotropic structure, with a linear (in pressure) variation in the vertical, and a linear variation in the horizontal along $-\hat{j}$ :

$T^{\prime}(y, p)=A \frac{p}{p_{s}}\left(\frac{y_{0}-y}{L}\right)$

such that the temperature perturbation varies by an amount $A$ over a distance $L$ near the surface. The parameter $p_{s}$ corresponds to a representative surface pressure value and $y_{0}$ is the meridional location of the domain centre. This gives the meridional temperature gradient

$\vec{\nabla} T^{\prime}(p)=-\frac{A}{L} \frac{p}{p_{s}} \hat{j}$

which can be integrated in the vertical to give the thermalwind relationship

$u^{\prime}\left(p_{s}\right)-u^{\prime}(p)=-\frac{R A}{f L p_{s}} \int_{p}^{p_{s}} p \partial \ln p$

Assuming $u^{\prime}\left(p_{s}\right)=0$ for simplicity gives

$u^{\prime}(p)=\frac{R A}{f L p_{s}}\left(p_{s}-p\right)$

We can now calculate the domain integrals of $A_{I V}$ and $K_{I V}$ as follows:

$$
\begin{aligned}
\left\{A_{I V}\right\} & =\frac{C_{p}}{2 T_{r}}\left\{T^{\prime 2}\right\} \\
\left\{K_{I V}\right\} & =\frac{1}{2}\left\{u^{\prime 2}\right\} \\
\left\{A_{I V}\right\} & =\frac{C_{p}}{2 T_{r}}\left\{T^{\prime 2}\right\} \\
& =\frac{C_{p}}{2 T_{r}} \int_{y=y_{0}-L / 2}^{y_{0}+L / 2} \int_{p=0}^{p_{s}}\left[A \frac{p}{p_{s}}\left(\frac{y_{0}-y}{L}\right)\right]^{2} d p d y /\left(p_{s} L\right) \\
& =\frac{C_{p} A^{2}}{2 T_{r} p_{s}^{3} L^{3}} \int_{y=y_{0}-L / 2}^{y_{0}+L / 2} \int_{s}^{p_{s}}\left[p\left(y_{0}-y\right)\right]^{2} d p d y \\
& =\frac{C_{p} A^{2}}{2 T_{r} p_{s}^{3} L^{3}} \frac{p_{s}^{3}}{3} \frac{2(L / 2)^{3}}{3} \\
& =\frac{C_{p} A^{2}}{72 T_{r}}
\end{aligned}
$$




$$
\begin{aligned}
\left\{K_{I V}\right\} & =\frac{1}{2}\left\{u^{\prime 2}\right\} \\
& =\frac{1}{2} \int_{y=y_{0}-L / 2}^{y_{0}+L / 2} \int_{p=0}^{p_{s}}\left[\frac{R A}{f L p_{s}}\left(p_{s}-p\right)\right]^{2} d p d y /\left(p_{s} L\right) \\
& =\frac{1}{2} \int_{p=0}^{p_{s}}\left[\frac{R A}{f L p_{s}}\left(p_{s}-p\right)\right]^{2} d p / p_{s} \\
& =\frac{R^{2} A^{2}}{2 f^{2} L^{2} p_{s}^{3}} \frac{p_{s}^{3}}{3} \\
& =\frac{R^{2} A^{2}}{6 f^{2} L^{2}}
\end{aligned}
$$

so that

$\frac{\left\{K_{I V}\right\}}{\left\{A_{I V}\right\}}=\frac{\frac{R^{2} A^{2}}{6 f^{2} L^{2}}}{\frac{C_{p} A^{2}}{72 T_{r}}}=\frac{12 R^{2} T_{r}}{C_{p} f^{2} L^{2}}$

Using representative values for mid-latitude weather disturbances, $L=20^{\circ} \approx 2200 \mathrm{~km}=2.2 \times 10^{6} \mathrm{~m}$, with $f=10^{-4} \mathrm{~s}^{-1}, T_{r}=250 \mathrm{~K}, R=287 \mathrm{~m}^{2} \mathrm{~K}^{-1} \mathrm{~s}^{-2}$, $C_{p}=1004 \mathrm{~m}^{2} \mathrm{~K}^{-1} \mathrm{~s}^{-2}$ gives: $\frac{\left\{K_{I V}\right\}}{\left\{A_{I V}\right\}}=5.1$.

\section{References}

Alexandru A, de Elia R, Laprise R (2007) Internal Variability in regional climate downscaling at the seasonal scale. Mon Weather Rev 135:3221-3238

Caya D, Biner S (2004) Internal variability of RCM simulations over an annual cycle. Clim Dyn 22:33-46

Caya D, Laprise R (1999) A semi-implicit semi-Lagrangian regional climate model: the Canadian RCM. Mon Weather Rev 127:341-362

Christensen OB, Gaertner MA, Prego JA, Polcher J (2001) Internal variability of regional climate models. Clim Dyn 17:875-887

Giorgi F, Bi X (2000) A study of internal variability of regional climate model. J Geophys Res 105:29503-29521

Hernández-Díaz L, Laprise R, Sushama L, Martynov A, Winger K, Dugas B (2013) Climate simulation over CORDEX Africa domain using the fifth-generation Canadian Regional Climate Model (CRCM5). Clim Dyn 40:1415-1433 doi:10.1007/s00382-012-1387-z, http:// link.springer.com/article/10.1007\%2Fs00382-012-1387-z

Kalnay E et al (1996) The NCEP-NCAR 40-year reanalysis project. Bull Am Meteorol Soc 77:437-471
Lorenz EN (1955) Available potential energy and the maintenance of the general circulation. Tellus 7:157-167

Lorenz EN (1963) Deterministic nonperiodic flow. J Atmos Sci 20:130 141. doi:http://dx.doi.org/10.1175/1520-0469(1963)020<0130: $\mathrm{DNF}>2.0 . \mathrm{CO} ; 2$

Lorenz EN (1967) The nature and theory of the general circulation of the atmosphere. World Meteorological Org. 218 TP $115161 \mathrm{pp}$

Lucas-Picher P, Caya D, Biner S (2004) RCM's internal variability as function of domain size. Research activities in atmospheric and oceanic modelling. WMO/TD, J Côté Ed, 1220 34:7.27-7.28

Lucas-Picher P, Caya D, de Elía R, Laprise R (2008a) Investigation of regional climate models' internal variability with a ten-member ensemble of ten-year simulations over a large domain. Clim Dyn 31:927-940. doi:10.1007/s00382-008-0384-8

Lucas-Picher P, Caya D, Biner S, Laprise R (2008b) Quantification of the lateral boundary forcing of a regional climate model using an ageing tracer. Mon Weather Rev 136:4980-4996

Marquet P (1991) On the concept of exergy and available enthalpy: application to atmospheric energetics. Q J R Meteorol Soc 117:449-475

Marquet P (2003a) The available-enthalpy cycle. I: introduction and basic equations. Q J R Meteorol Soc 129(593):2445-2466

Marquet P (2003b) The available-enthalpy cycle. II: applications to idealized baroclinic waves. Q J R Meteorol Soc 129(593):2467-2494

Martynov A, Laprise R, Sushama L, Winger K, Šeparović L, Dugas B (2013) Reanalysis-driven climate simulation over CORDEX North America domain using the Canadian Regional Climate Model, version 5: Model performance evaluation. Clim Dyn 41(11-12):2973-3005 http://link.springer.com/article/10.1007/ s00382-013-1778-9

Nikiéma O, Laprise R (2011a) Diagnostic budget study of the internal variability in ensemble simulations of the Canadian RCM. Clim Dyn 36(11): 2313-2337. doi:10.1007/s00382-010-0834-y, http:// www.springerlink.com/content/bn3n5321514002q6/fulltext.pdf

Nikiéma O, Laprise R (2011b) Budget study of the internal variability in ensemble simulations of the Canadian RCM at the seasonal scale. J Geophys Res Atmos. 116(D16112). doi:10.1029/2011JD015841, http://onlinelibrary.wiley.com/doi/10.1029/2011JD015841/full

Nikiéma O, Laprise R (2013) An approximate energy cycle for intermember variability in ensemble simulations of a regional climate model. Clim Dyn 41:831-852. doi:10.1007/s00382-012-1575-x

Pearce RP (1978) On the concept of available potential energy. Q J R Meteorol Soc 104:737-755

Rinke A, Dethloff K (2000) On the sensitivity of a regional Arctic climate model to initial and boundary conditions. Clim Res 14:101-113

Rinke A, Marbaix P, Dethloff K (2004) Internal variability in Arctic regional climate simulations: case study for the Sheba year. Clim Res 27:197-209

Weisse R, Heyen H, von Storch H (2000) Sensitivity of a regional atmospheric model to a sea state dependent roughness and the need of ensemble calculations. Mon Weather Rev 128:3631-3642 